\title{
The VHITAL Program to Demonstrate the Performance and Lifetime of a Bismuth-Fueled Very High Isp Hall Thruster
}

\author{
Colleen Marrese-Reading ", Anita Sengupta*, Robert Frisbee*, Jay Polk ${ }^{\dagger}$, \\ Jet Propulsion Laboratory, California Institute of Technology, Pasadena, CA, 91109 \\ Mark Cappelli $i^{\ddagger}$ \\ Stanford University, Stanford, CA 94305 \\ Iain Boyd ${ }^{\S}$ and Michael Keidar** \\ University of Michigan, Ann Arbor, MI, 48109-2140 \\ Sergey Tverdokhlebov ${ }^{\dagger+}$ and Sasha Semenkin ${ }^{\ddagger \ddagger}$ \\ Central Scientific Research Institute of Machine Building (TsNIIMASH), Korolev, Russia \\ Tom Markusic $\$ \$$ \\ NASA Marshall Space Flight Center, Huntsville, AL, 35812 \\ Azer Yalin ${ }^{* * *}$ \\ Colorado State University, Fort Collins, CO, 80523-1374 \\ and \\ Timothy Knowles ${ }^{+\dagger+}$ \\ Energy Science Laboratories, Inc., San Diego, CA, 92121
}

\begin{abstract}
In the Very High Isp Thruster with Anode Layer (VHITAL) Program the performance, plume and lifetime capability of the radiatively-cooled two stage, bismuth-fueled VHITAL160 will be characterized in the U.S. and Russia. The demonstration and further characterization of this technology is motivated by its excellent performance, unique propellant attributes and potentially significant mass and trip time savings over chemical and ion thruster systems for NEP missions. This Russian-developed electric propulsion technology will be improved from its previously tested state over 25 years ago with an advanced feed system, novel erosion and plume diagnostics and models of the thruster performance, lifetime and plume interactions with the spacecraft. The VHITAL-160 design documentation has been completed with thermal and structural analysis showing the capability of this thruster to operate in a self-heated mode at 25 and $36 \mathrm{~kW}$. The previously existing TAL-160 was refurbished at TSNIMASH and recently demonstrated operation in a discharge mode and the functionality of the thruster support systems. An all-carbon
\end{abstract}

\footnotetext{
${ }^{*}$ Senior Engineer, Advanced Propulsion Technology, M/S 125-109, 4800 Oak Grove Dr.

† Principal Engineer, Advanced Propulsion Technology, M/S 125-109, 4800 Oak Grove Dr.

${ }^{\ddagger}$ Professor, Department of Mechanical Engineering

${ }^{\$}$ Professor, Department of Aerospace Engineering, 3012 FXB, 1320 Beal Ave.

${ }^{* *}$ Assistant Research Scientist, Department of Aerospace Engineering, FXB, 1320 Beal Ave.

${ }^{+\dagger}$ JSC TsNIIMASH-Export, Executive Director

$\stackrel{++}{++}$ Director of Electric Propulsion Laboratory, TsNIIMASH

Senior Engineer, Propulsion Research Center Bldg. 4205

${ }^{* * *}$ Assistant Professor, Department of Mechanical Engineering

${ }^{+++}$President, 6888 Nancy Ridge Dr.
} 
resistively heated prototype vaporizer with porous plug was successfully demonstrated for the flight-like feed system. Hydrodynamic models of the first and second stages of the discharge have been developed with good agreement with experimental results. Significant progress has been made in the development and demonstrations of the thruster diagnostics.

\section{Introduction}

The bismuth-fueled two stage thruster with anode layer technology is being resurrected because of its unique power, specific impulse, performance capabilities and propellant attributes that are attractive for NASA missions. This propulsion technology is attractive for NEP missions to the outer planets and SEP Mars and lunar cargo missions because it has demonstrated excellent performance (60-80\%) at $2000-8000$ s specific impulse at $10-100$ $\mathrm{kW}$ per thruster. These two stage anode layer thrusters were developed at TsNIIMASH more than 25 years ago. The water-cooled TAL- 160 demonstrated up to $140 \mathrm{~kW}$ and $8000 \mathrm{~s}$. The radiatively-cooled TAL-200 demonstrated 10 $34 \mathrm{~kW}$ at $2000-5200 \mathrm{~s}$.

This thruster technology has several advantages for high power operation over the conventional single stage Hall thrusters including a primarily robust metal construction, the two stage configuration for higher specific impulse operation, smaller size for higher thrust density, a propellant that has a much lower cost than xenon and is much less expensive to pump in ground test facilities. The maximum specific impulse that advanced single stage Hall thrusters have demonstrated is $3700 \mathrm{~s}$ with the SPT-1[1]. At voltages greater than $1 \mathrm{kV}$, the single stage Hall thrusters exhibit a decrease in efficiency. This limitation results from a fundamental change in the behavior of the electrons in the discharge chamber at such high voltages. Deleterious anode heating occurs as the energy of the electrons collected by the anode and the electron current to the anode increase with increases in the voltage applied for ionization and ion acceleration. The maximum achievable ion velocity is, therefore, limited by the thermal constraints for the anode. This problem is solved in a two stage TAL that separates the ionization and acceleration regions and decouples anode heating from the accelerating voltage. The high specific impulse necessary for potential NEP missions to the outer planets $(6000$ to $9000 \mathrm{~s})$ requires ion accelerating voltages greater than $4 \mathrm{kV}$. Two stage TALs have demonstrated increasing efficiency with operating voltage through $8 \mathrm{kV}[2]$.

The abundantly available bismuth propellant is very attractive as a propellant for high power propulsion systems. The most significant advantages to using a bismuth-fueled thruster over a thruster that uses xenon or even lithium are identified in Table 1. For a mission requiring $10,000 \mathrm{~kg}$ of propellant, the propellant could cost $\$ 20.0 \mathrm{M}$ for xenon and only $\$ 0.75 \mathrm{M}$ for bismuth. The cost difference also represents a significant savings for ground testing. The melting temperature of bismuth, at $271^{\circ} \mathrm{C}$, enables it to condense at room temperature on vacuum facility walls. This attribute of the propellant significantly

Table 1. Characteristics of bismuth propellant which make it an attractive alternative to conventional electric propulsion system propellants.

\begin{tabular}{|l|c|c|c|}
\hline Properties & Bismuth & Xenon & Lithium \\
\hline $\begin{array}{l}\text { Density }\left(\mathrm{kg} / \mathrm{m}^{3}\right) \\
\text { at } 20^{\circ} \mathrm{C}\end{array}$ & $\begin{array}{c}9780 \\
\text { (solid) }\end{array}$ & $\begin{array}{c}2000 \\
(2800 \mathrm{PSI} \\
\left.40^{\circ} \mathrm{C}\right)\end{array}$ & $\begin{array}{c}535 \\
\text { (solid) }\end{array}$ \\
\hline Cost $(\$ / \mathrm{kg})$ & 75 & 2000 & 137 \\
\hline $\begin{array}{l}\text { lonization } \\
\text { Potential }(\mathrm{eV})\end{array}$ & 7.29 & 12.12 & 5.39 \\
\hline
\end{tabular}

relaxes the pumping speed requirements placed on facilities used for testing bismuth fueled thrusters, which is a significant factor when testing at increasingly high power levels for high power electric thruster performance and lifetime assessments. Two stage TALs operating on Bi propellant could be tested at power levels exceeding $100 \mathrm{~kW}$ in existing facilities. Multiple thrusters could be tested in existing facilities at total power levels exceeding $1 \mathrm{MW}$. Additional attributes of the bismuth propellant include its low ionization potential and high mass, enabling high efficiency thruster operation. It is also environmentally friendly and non-toxic.

The VHITAL Program P-I is Dr. Mark Cappelli of Stanford University. He is also leading the thruster lifetime assessment element. He is responsible for implementing the near-field plume diagnostics and leading the near-field plume characterization and the assessment of the thruster lifetime. Anita Sengupta, a Co-I, is the VHITAL Program Manager at JPL. Dr. Colleen Marrese-Reading is a Co-l as the technical lead of the thruster support system technology development and the thruster performance assessment at JPL. Drs. Sergey Tverdokhlebov and Sasha Sememkin, at TsNIIMASH, are responsible for the thruster, cathode and a feed system. Dr. lain Boyd, at the University of Michigan, is also a Co-l who will lead the spacecraft contamination- assessment element. He is responsible for modeling the internal, near-field and far-field flow and determining the characteristies of the plasma that will interact with the spacecraft systems. Dr. Kevin Rudolph (Co-I), at Lockheed Martin Astronautics 
Operations, will be lead the spacecraft system analysis element. He will be responsible for system analysis, focusing on the optimal operating conditions and thruster system configurations to provide the best architectures for NEP missions. Dr. John Williams and Dr. Azare Yalin at Colorado State University are participating in the thruster lifetime assessment through erosion product flux and guard ring material sputter yield measurements. Dr. Jay Polk will lead the SLA diagnostic implementation to measure the thruster erosion rates. Dr. Lee Johnson and Dr. David Conroy will assist in the implementation of the lifetime and plume diagnostics at JPL. Dr. Tom Markusic, at NASA MSFC, is responsible for the propellant flow rate sensor and the propellant pump.

The comprehensive program includes both experimental performance evaluations and theoretical performance modeling, the development of a three-pronged approach to experimental life assessment, guard ring material sputter yield measurements, theoretical life modeling to evaluate the thruster lifetime beyond the scope of the experimental program, plume diagnostics and the development of a plume model to predict the potential deposition rate of bismuth on spacecraft surfaces. A two pronged approach is being pursued to control and measure the mass flow rate of bismuth to the thruster including a system that was demonstrated in previous testing of the TAL 160 and TAL 200. The erosion rate model and data will be applied to enable lifetime assessments beyond the resources of the experimental program.

The 2.5 year VHITAL Program started with the six month phase one in July 2004 through December 2004. The six month first phase of the program focused on program planning, thruster and support system design, TAL-160 refurbishment, thruster design analysis diagnostic approach development and modeling approach development. The one year second phase of the program started in February 2005. In this phase of the program the thruster design documentation has been completed, a previously existing TAL-160 will be used to demonstrate the operability of the VHITAL-160 support systems, all of the support systems will be demonstrated, the diagnostics will be developed, the thruster performance and plume model will progress significantly and the VHITAL 160 will be built and acceptance tested at TsNIIMASH. In the one year phase three the thruster will be delivered to JPL where performance, lifetime and plume characterizations will be conducted at the critical operating points. Sputter yields of guard ring materials will be measured. The lifetime and plume models will be further developed with integrated experimental data and applied to predict thruster lifetime and spacecraft contamination potential

\section{A Two Stage Hall Thruster with Anode Layer Operation}

In a two stage TAL, the ion generation region is separated from the acceleration region to limit back-streaming electron current through the accelerating layer. This configuration maximizes the accelerating efficiency and minimizes the power used for ionization. A VHITAL design optimizes the split of ionization between the acceleration and ion production regions to maximize overall efficiency. Anode power dissipation in the VHITAL is expected to be approximately $25 \%$ of the anode power dissipation in a single-stage thruster at the same operating conditions. The two-stage configuration enables effective ionization at current densities much lower than that in the single-stage devices. This capability leads to longer lifetime because current density has a first-order impact on thruster wear mechanisms.

The thruster employs a magnetic system similar to traditional Hall device designs with inner and outer pole pieces and an inner electromagnet. A rough schematic of a two-stage TAL developed by TsNIIAMSH is shown in Figure 1. The VHITAL magnetic field topography minimizes the inclination of the ion

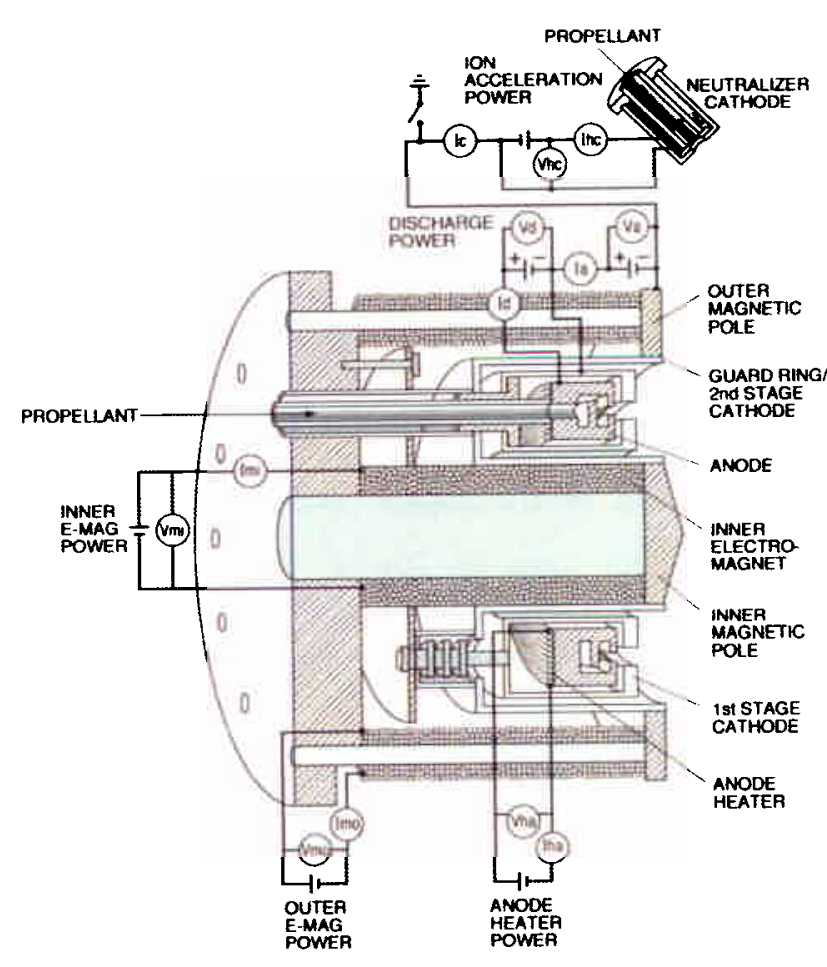

Figure 1. Schematic of a two stage TAL. 
beam from the thruster axis to improve thruster efficiency.

The first stage (low-voltage stage) is comprised of the annular anode distributor and a ring cathode. To provide anode pre-heating for operation with condensable propellant, the anode is equipped with a special ring heater located inside the anode body. The anode is fabricated from refractory metals because of the necessity to keep the anode-distributor at the temperature $\left(1000^{\circ} \mathrm{C}\right)$ corresponding to a $\mathrm{Bi}$ saturation pressure of about $500 \mathrm{~Pa}$ (3.7 Torr).

The second stage (high-voltage stage) is formed by the ring cathodes of the first stage and the guard rings positioned at the downstream end of the thruster. These guard rings protect the magnet pole pieces from sputtering, but are themselves subject to sputter erosion. The cathode material for the first and second stages (i.e., the guard rings) must also have a high melting temperature (exceeding $1500^{\circ} \mathrm{C}$ ) and good radiating characteristics at high temperature.

Both the first-stage and second-stage electrodes are electrically isolated from the thruster body and from each other with ceramic insulators. The electrode assembly is installed inside the magnetic system on a mounting flange, providing additional thermal insulation of the electromagnets from the discharge chamber elements. This flange, the magnet system, and guard rings are maintained at the same electric potential as the cathode-neutralizer installed near the thruster exit plane.

Evaporated $\mathrm{Bi}$ propellant is supplied to the anode for azimuthal distribution in the discharge chamber. Ionization occurs in the first stage, where a low discharge voltage (typically 150 to $250 \mathrm{~V}$ ) is sustained. The ions are accelerated in the second stage by the accelerating voltage applied between the guard rings and the first-stage cathode (several kilovolts). While the optimal discharge voltage in the first stage is only slightly sensitive to operating conditions, the accelerating voltage is regulated over the range required to produce the desired $I s p$. Space charge and current neutralization of the ion beam is provided by the external cathode.

\section{The VHITAL 160}

The radiatively-cooled VHITAL 160 was designed by TsNIIMASH. It is a radiatively-cooled version of the watercooled TAL-160 that was developed over 25 years ago and demonstrated up to $140 \mathrm{~kW}$. The performance and lifetime of the VHITAL-160 will be characterized at $25(6000 \mathrm{~s})$ and $36 \mathrm{~kW}$ $(8000 \mathrm{~s})$. The thruster design documentation was completed and a CAD drawing of the thruster is shown in Figure 2. The two critical operating points are described in Table 2. The previously developed and recently refurbished TAL-160 is shown in Figure 3. It was refurbished under this program to demonstrate all of the support systems before testing the VHITAL-160.

Thermal and structural analysis were conducted on the VHITAL 160 design at TsNIIMASH. The 3-D thermal model was used to estimate the thruster component temperatures at 25 and $36 \mathrm{~kW}$ both with an anode heater and in a self-heated mode. The results showed that the temperatures of the thruster components are all within the tolerable regimes for the materials

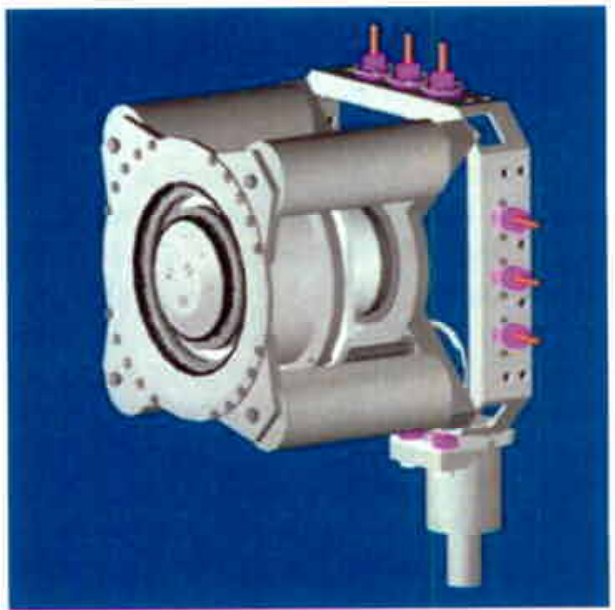

Figure 2. A CAD drawing of the VHITAL 160 with feed system.

selected. Temperatures of the anode and the two cathode electrodes will be higher than the bismuth condensation temperatures during preheating and thruster operation. The thruster design meets the requirement of the complete radiative cooling scheme for the thruster for both the 25 and $36 \mathrm{~kW}$ operating points. Expected temperatures of the thruster components will not compromise the structural integrity of the thruster. The results also confirmed the capability of the thruster to operate at both 25 and $36 \mathrm{~kW}$ in a self-heated mode. The structural analysis was conducted with the results of the thermal analysis. The results showed that, at high temperatures, the electrodes will 
expand along the thruster axis without increased mechanical stress, distortion or misalignment. They also showed that the required clearances were maintained even at the maximum expected expansion of the highest temperature elements to eliminate the possibility of electrical shorts.

Hollow cathode neutralizers will be used as the electron source for the thruster. TsNIIMASH will provide a bismuth fueled $\mathrm{LaB}_{6}$ cathode. The cathode design was demonstrated using bismuth propellant in previous programs. JPL will provide a xenon fueled hollow cathode which is identical to the 0.635 -cm hollow cathode employed in the NSTAR ion thruster discharge chamber. For VHITAL operation at 20 to $36 \mathrm{~kW}$, a cathode current of approximately 4-5 $\mathrm{A}$ is required. The NSTAR hollow cathode operates on Xe propellant, and is capable of providing 4 to $16 \mathrm{~A}$ of discharge current. This cathode will employ a 4:1:1 Barium Calcium Aluminate impregnated tungsten thermionic emitter as the electron source. Utilizing a Xe-fueled hollow cathode is advantageous as it reduces the spacecraft contamination potential of the VHITAL 160 thruster. Xe collisions with high velocity $\mathrm{Bi}$ ions produce significantly fewer high angle ions than $\mathrm{Bi}-\mathrm{Bi}$ ion collisions, due to the unequal mass interaction. Analysis shows that the Bi-Xe elastic scattering angle will never exceed 39 degrees, whereas a $\mathrm{Bi}-\mathrm{Bi}$ elastic scattering angle of 90 degrees may occur. Analysis shows that inelastic charge exchange interaction can produce high angle scattering, however, in the case of Xe propellant the Bill-XeI charge exchange production can be assumed

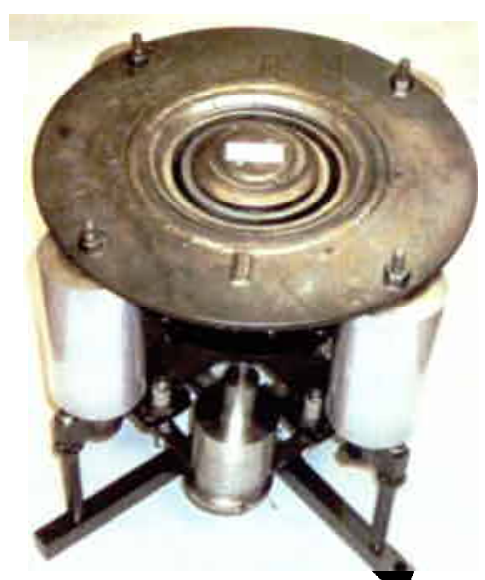

Figure 3. The refurbished TAL-160. to be negligible as the ionization potential of $\mathrm{Xe}$ is $5 \mathrm{eV}$ greater than that of $\mathrm{Bi}$. It is also assumed that the beam plasma of the TAL is greater than $90 \%$ ionized, and that the only source of neutral propellant is the neutralizer cathode for either a Bi or Xe fed neutralizer.

\section{Mission Studies}

This section of the paper presents the results of mission analyses that expose the advantages of the Bismuth (Bi) propellant Very High $\mathrm{I}_{\mathrm{sp}}$ Thruster with Anode Layer (VHITAL) electric propulsion system for missions considered under Prometheus. The analyses include comparison studies of the VHITAL technology relative to state-of-the-art Ion propulsion systems and quantify the unique benefits of the technology such as high power-per-thruster, high specific impulse, and low tankage mass due to the high density of bismuth propellant. NEP robotic missions and Mars cargo missions were considered in this study. The NEP robotic missions include interstellar precursor missions at $5 \mathrm{AU} / \mathrm{yr}$ and $10 \mathrm{AU} / \mathrm{yr}$ and a mission to Saturn with a moon tour.

\section{A. MISSION ANALYSIS RESULTS FOR NEP ROBOTIC PLANETARY MISSIONS}

\section{Nuclear Electric Propulsion Robotic Planetary Vehicle Assumptions}

The NEP vehicle consists of a nuclear-electric power system, a main boom that is used both to support the power system's radiators and to separate the spacecraft systems and payload from the reactor's radiation, a power management and distribution (PMAD) system (high-power cabling between the power system and the spacecraft
bus), and the spacecraft bus and payload. The spacecraft bus contains the reaction control system (RCS), various miscellaneous spacecraft systems (e.g. telecommunications, etc.), and the electric propulsion system.

\section{NEP Vehicle Configuration}

A conceptual schematic of the NEP vehicle is shown in Figure 4. Note that some electric propulsion options may require the use of a plume shield to protect sensitive spacecraft surfaces from the thrusters' exhaust plume. For example, spacecraft surfaces can be subjected to thermal contamination or physical erosion from the high-energy plume. Also, there is the risk of material contamination by condensable propellants, such as the bismuth used in the VHITAL thruster. Material contamination can be a serious concern for optically-sensitive systems like camera lenses, and especially for radiators where the contaminant material can change the radiator's emissivity.
Also, placing the electric thrusters at the far end of the vehicle, with a $180^{\circ}$ field of view to space, ensures that no spacecraft surfaces will experience thermal contamination or erosion impact from the thruster plumes. Fortunately, plume contamination with VHITAL can be minimized by use of a plume shield. Thus, we included a $10-\mathrm{m}$ diameter plume shield in our analyses of both NEP vehicles using VHITAL thrusters. 


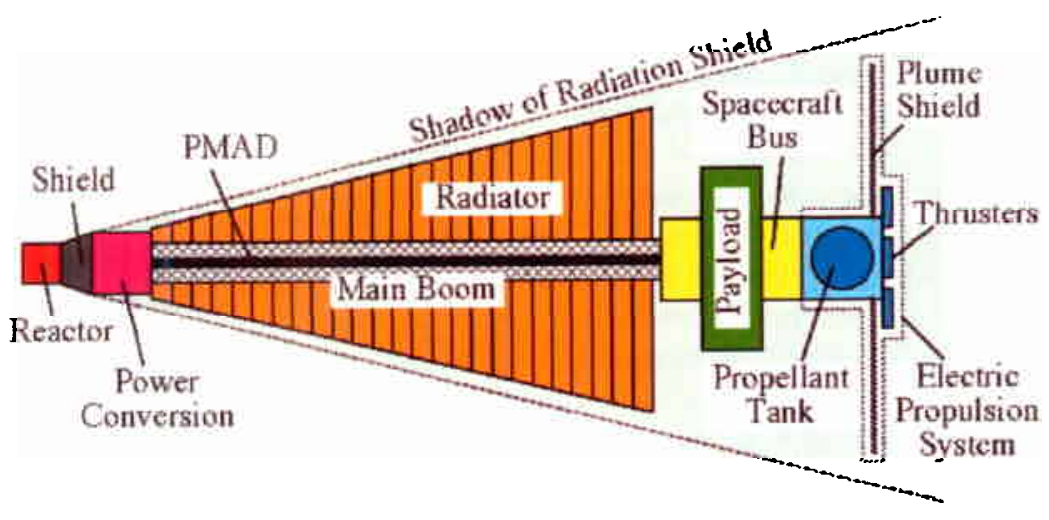

Figure 4. Conceptual Schematic of an NEP Vehicle.

\section{Nuclear Electric Power System}

Mass scaling estimates for the nuclear-electric power system were provided by Lee Mason (NASA Glenn Research Center, GRC). The power system consists of a reactor, shield, heat exchanger, dynamic (Brayton) thermalto-electric power conversion system, and waste heat rejection system (radiators, pumps, fluid loops, etc.). The overall system specific mass $\left(\mathrm{kg} / \mathrm{kW}_{\mathrm{e}}\right)$ is shown in Figure 5 as a function of total or "bus" power level. Also shown are the specific masses of the main boom and PMAD systems. As is commonly observed in space-based NEP power systems, there is a significant economy-of-scale at higher powers. This often tends to drive the power requirement to high power levels in order to achieve short trip times, because the overall vehicle specific mass is inversely proportional to overall vehicle acceleration for a given $I_{\text {sp }}$. Also, higher powers reduce the effective specific mass of fixed-mass vehicle elements (e.g., payload), again favoring increased power for short trip times.

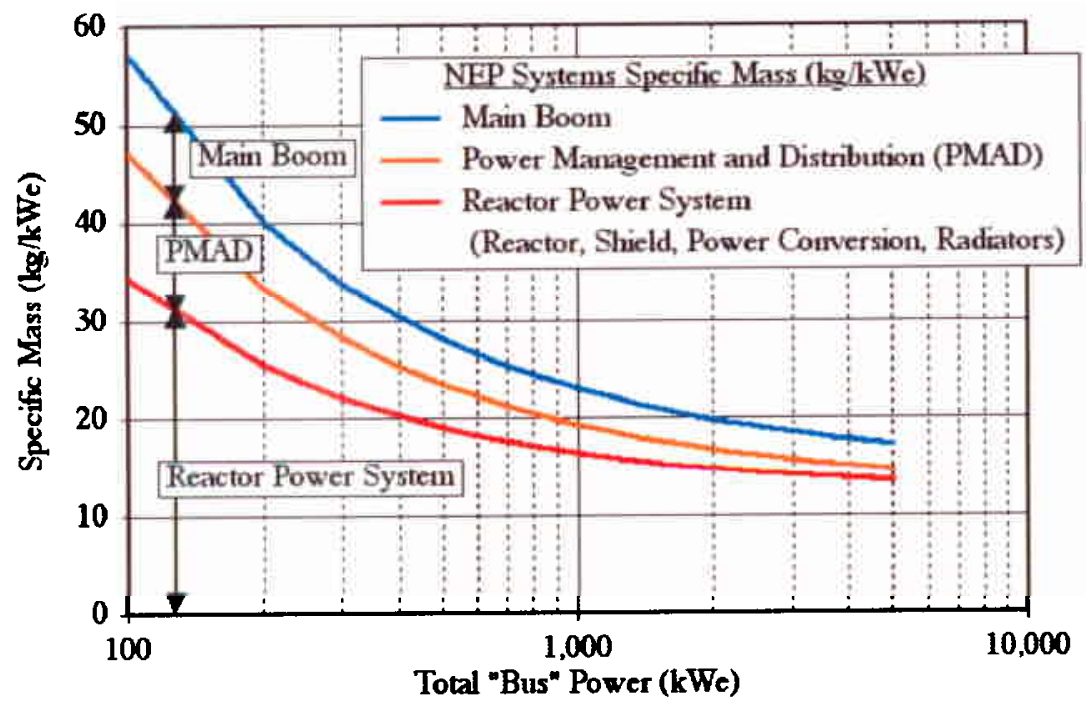

Figure 5. NEP Power System, Main Boom, and Power Management and Distribution (PMAD) System Specific mass as a Function of Total "Bus" Power.

\section{Main Boom}

In an NEP vehicle, the main boom runs from the power system to the main spacecraft bus, as illustrated in Figure 4. The boom serves to both support the power system's radiators, as well as provide standoff distance to reduce the radiation load on the spacecraft systems and payload. Mass scaling estimates for the main boom were provided by Muriel Noca (NASA Jet Propulsion Laboratory, JPL). The mass of the main boom includes the forward (power-system end) equipment structure that attaches the boom system to the power system, the boom deployment canister (including drive motors, actuators, etc.), the boom structure itself, its mounting structure, cabling (PMAD) 
attachment hardware, thermal control, and micrometeoroid protection. As shown in Figure 5, there is only a slight economy-of scale due to the need to strengthen the boom structure to prevent buckling as the boom length increases with increased power.

For calculation purposes, the mass per unit length of the main boom was calculated based on the radiator length, which in turn is a function of the radiation shield's shadow half-angle (assumed to be $10^{\circ}$ ). An additional 10-m length of boom was then added to represent separation distance between the hot radiator and the spacecraft bus.

\section{Power Management and Distribution (PMAD)}

The power management and distribution (PMAD) system consists of high-power cabling between the power system and the spacecraft bus. Mass scaling estimates for the NEP PMAD system were provided by Lee Mason (NASA GRC). As with the main boom, the mass per unit length of the PMAD cabling was estimated based on the boom length, and an additional $10 \mathrm{~m}$ of PMAD was added to correspond to the extra $10 \mathrm{~m}$ of boom length.

As shown in Figure 5, there can be a substantial economy of scale for the PMAD system. Also note that in an NEP vehicle, the electric power produced by the turboalternators in the dynamic power system is in the form of high-voltage AC. By contrast, the electric power from the photovoltaic arrays in an SEP vehicle is in the form of relatively low-voltage DC. Thus, for a given power level, the PMAD for an SEP "bus" will be somewhat heavier than for an NEP system, although the mass penalty for a low-voltage system is somewhat offset by the additional electrical insulation and isolation required for a high-voltage system. In fact, the greatest PMAD impact is in the electric propulsion system power processing units (PPUs) that convert the "bus" voltage to the form required by the electric thruster. For example, an Ion or Hall (VHITAL) thruster requires very high-voltage (typically many $\mathrm{kV}$ ) DC. Thus, the PPU for an NEP system requires only a transformer (to convert the "bus" voltage level to that required by the thruster) and rectifiers (to convert the "bus" AC to DC). By contrast, a PPU for an SEP vehicle requires an additional initial DC-to-AC invertor, along with a transformer and rectifier as in the NEP PPU. The topology of these various options is illustrated in Figure 6. Thus, an SEP-PPU is generally heavier than an NEPPPU.

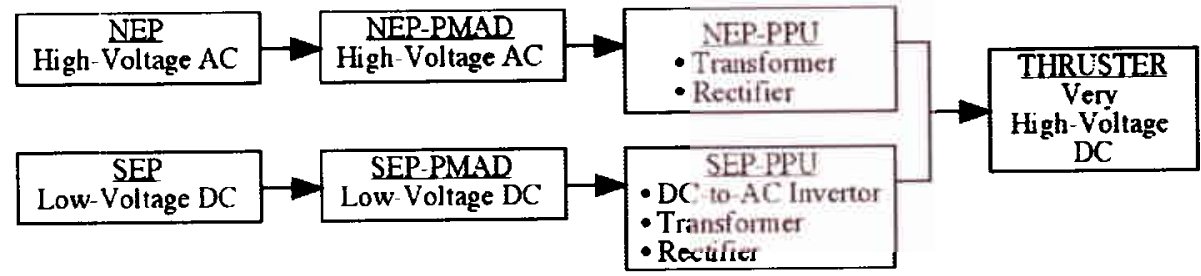

Figure 6. NEP and SEP Power System, PMAD, PPU, and Thruster Topology.

\section{General Structural Mass Overhead, and Electric Power and Dry Mass Contingencies}

The power system, main boom, and PMAD masses include their own structural overhead. For the other various spacecraft systems described next, we have assumed a general structural overhead of $26 \%$ of the dry mass of the various components based on JPL system design study practices. Additionally, we have assumed a general structural overhead of $4 \%$ of the mass of propellants. These structural overheads represent miscellaneous structure required to tie the various components together and to the NEP velicle. Also, for those systems requiring electric power, we have assumed a power contingency of $10 \%$. The total power is subtracted from the total "bus" power in determining the power available to the electric propulsion system.

Finally, all dry masses (other than the payload) have an additional $30 \%$ dry mass contingency. This contingency, although large, is representative of current baselines for estimating spacecraft mass growth. Note that this is a relatively recent addition to mission analyses; many previous studies omitted this quantity.

\section{Chemical Hydrazine $\left(\mathbf{N}_{2} \mathrm{H}_{4}\right)$ Reaction Control System (RCS)}

A sequentially-recharged blowdown chemical hydrazine $\left(\mathrm{N}_{2} \mathrm{H}_{4}\right)$ reaction control system $(\mathrm{RCS})$ is used to maintain attitude control during all phases of the mission. The thrusters have an $\mathrm{l}_{\mathrm{sp}}$ of $220 \mathrm{lb}_{\mathrm{r}} \mathrm{s} / \mathrm{lb}_{\mathrm{m}}$; the mass of hydrazine is determined based on a $50-\mathrm{m} / \mathrm{s} \Delta \mathrm{V}$ for the fully loaded "wet" vehicle (with payload). The RCS components have a fixed mass of $18 \mathrm{~kg}$ and a tankage factor of $8.92 \%$ (including $0.73 \%$ for residuals and holdup). The RCS electric power requirement is $51 \mathrm{~W}_{\mathrm{e}}$ (primarily to power the hydrazine thruster catalyst bed heaters); a $10 \%$ margin is added to this for a total of $56 \mathrm{~W}_{\mathrm{e}}$. Finally, as described above, an overall structural factor of $26 \%$ is 
applied to the dry mass of the RCS (including tankage), and an additional $4 \%$ structure is applied to the mass of hydrazine propellant.

\section{Miscellaneous Spacecraft Systems}

A number of "generic" robotic spacecraft systems [3] are included in the NEP vehicle's mass. These systems include attitude control systems (ACS), command and data handling (C\&DH) systems, telecommunications (Telecom), vehicle cruise/emergency power (an allocation of $230 \mathrm{~kg}$ for some combination of batteries, radioisotope thermoelectric generators [RTGs] for deep-space missions, or solar arrays for inner Solar System missions), thermal control, and component-specific structure $(108 \mathrm{~kg}$ ). The total mass of the miscellaneous systems is $387 \mathrm{~kg}$ (without component-specific structure); $26 \%$ of this mass is added to represent miscellaneous structure, resulting in a total dry mass of $596 \mathrm{~kg}$. The total power is $328 \mathrm{~W}_{\mathrm{e}} ; \mathrm{a} 10 \%$ margin is added to this for a total of $361 \mathrm{~W}_{\mathrm{e}}$.

\section{Science Payload}

For these mission analyses, we have assumed a net science payload of $2,500 \mathrm{~kg}$ with a power requirement of 1 $\mathrm{kW}_{\mathrm{e}}$ during NEP operation and cruise. This represents a significant mass of payload that could include landers and probes for the Saturn Mission, or an independent, self-contained spacecraft for the Interstellar Precursor Mission that could be jettisoned after the NEP vehicle's propulsive burn to continue on its own out of the Solar System and beyond. Also, a structural adaptor of $2.5 \%$ of the payload's mass is added to tie the payload to the NEP vehicle. Additionally, the $30 \%$ dry mass contingency is added to the structural adaptor, but not the payload. Finally, a $10 \%$ margin is added to the payload's electric power requirement, such that the total power requirement is $1.1 \mathrm{~kW}_{\mathrm{e}}$.

\section{Ion (Herakles) Propulsion System}

The lon thruster used as a baseline for comparison with the NuPIT system is based on the Herakles thruster proposed for the JIMO mission. We developed a series of scaling equations to calculate mass, power, efficiency, etc. for this engine based on a theoretical model developed by Thomas Randolph (JPL), Doug Fiehler (QSS Group), and Kurt Hack (GRC).[4] The thruster characteristics assumed for our analyses are illustrated in Figure 7. The thruster mass is $58 \mathrm{~kg}$ (including an $8-\mathrm{kg}$ cable between the thruster and the PPU) with a beam diameter of about $69 \mathrm{~cm}$ (the overall outside diameter is roughly $94 \mathrm{~cm}$ ). (The flattening out of thruster power above an $I_{s p}$ of about $7,000 \mathrm{lb}_{\mathrm{f}}-\mathrm{S} / \mathrm{lb}_{\mathrm{m}}$ is due to limiting of the beam current density.) Finally, the Herakles thruster is assumed to have a propellant throughput of $5,500 \mathrm{~kg}$ of Xe propellant.

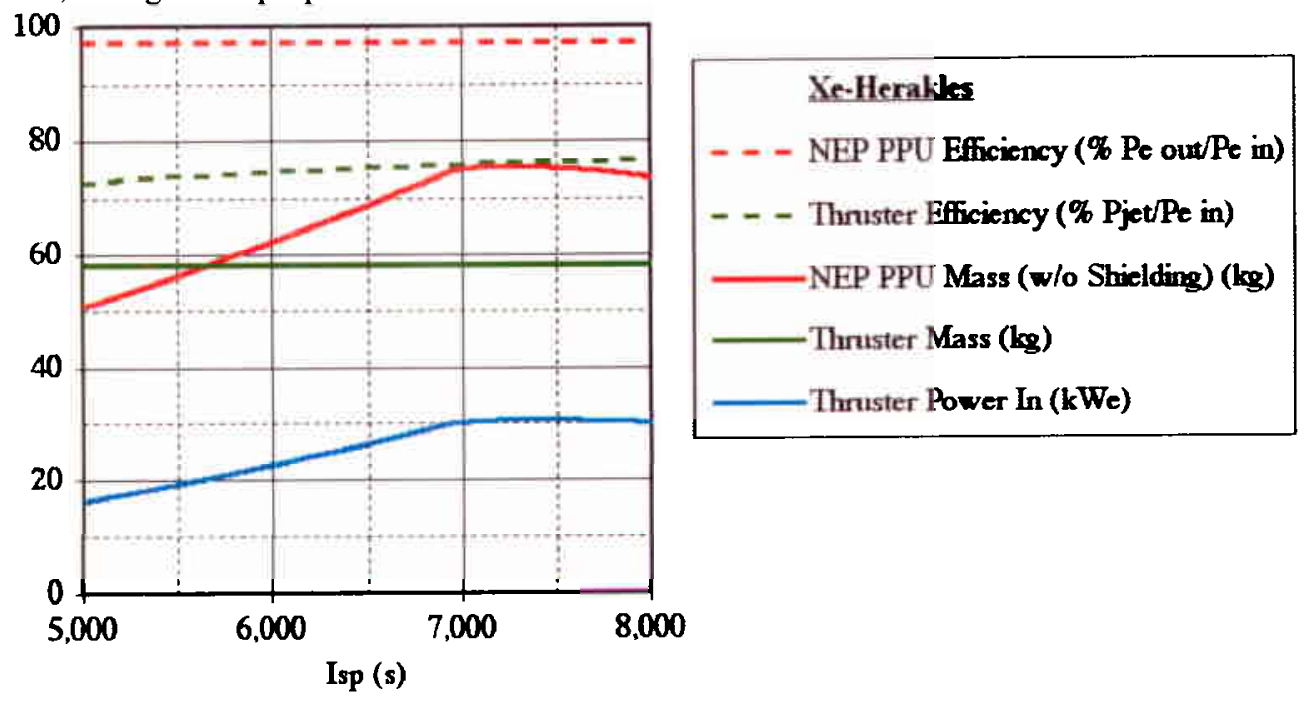

Figure 7. Ion (Herakles) Thruster and PPU Characteristics.

A mass list for a Xenon propellant storage and feed system was developed based on a design by Gani Ganapathi (NASA JPL). For the Xe-propellant lon thruster, we can store the propellant as high-pressure, room-temperature supercritical gas and feed the high-pressure Xe to the thrusters. Also, as shown in the feed system schematic below, a Xenon Recovery System (XRS) using a sorption compressor is used to scavenge residual Xe in the main tank as it nears depletion. This has the effect of dramatically reducing the amount of residual Xe that would otherwise be 
unavailable as pressure drops in the main tank. A schematic of the Xe-lon thruster propellant storage and feed system is shown in Figure 8. A summary of the various components is given in Table 3. Note that some electric heater power is required to maintain the system at room temperature to compensate for heat lost due to radiation in deep space far from the sun, as well as additional electric power corresponding to the heat of desorption of xenon in the Xenon Recovery System (XRS). Although this desorption power would only be needed near the end of the mission when the XRS is operating, we have assumed that it would be required at all times; thus, the various heater powers and XRS desorption power (equal to the XRS heat of desorption $[440 \mathrm{~J} / \mathrm{g}]$ multiplied by the mass flow rate $[\mathrm{g} / \mathrm{s}]$ of propellant into the lon thrusters) are subtracted from the total "bus" power. Also, note that there are both fixed-mass (or power) terms in the mass and power scaling relationships, as well as terms dependant on propellant mass $\left(M_{p}\right)$ and on surface area $\left(M_{p}{ }^{2,3}\right)$. Finally, a large number of ion thrusters are required on a $M_{\mathrm{e}}$-class vehicle. To facilitate vehicle integration and packaging in the launch vehicle, the Ion thrusters are collected into two clusters or "pods," with each pod having a gimbal and associated flex lines. The Ion thrusters are mounted directly on the pod structure (i.e., not individually gimbaled).
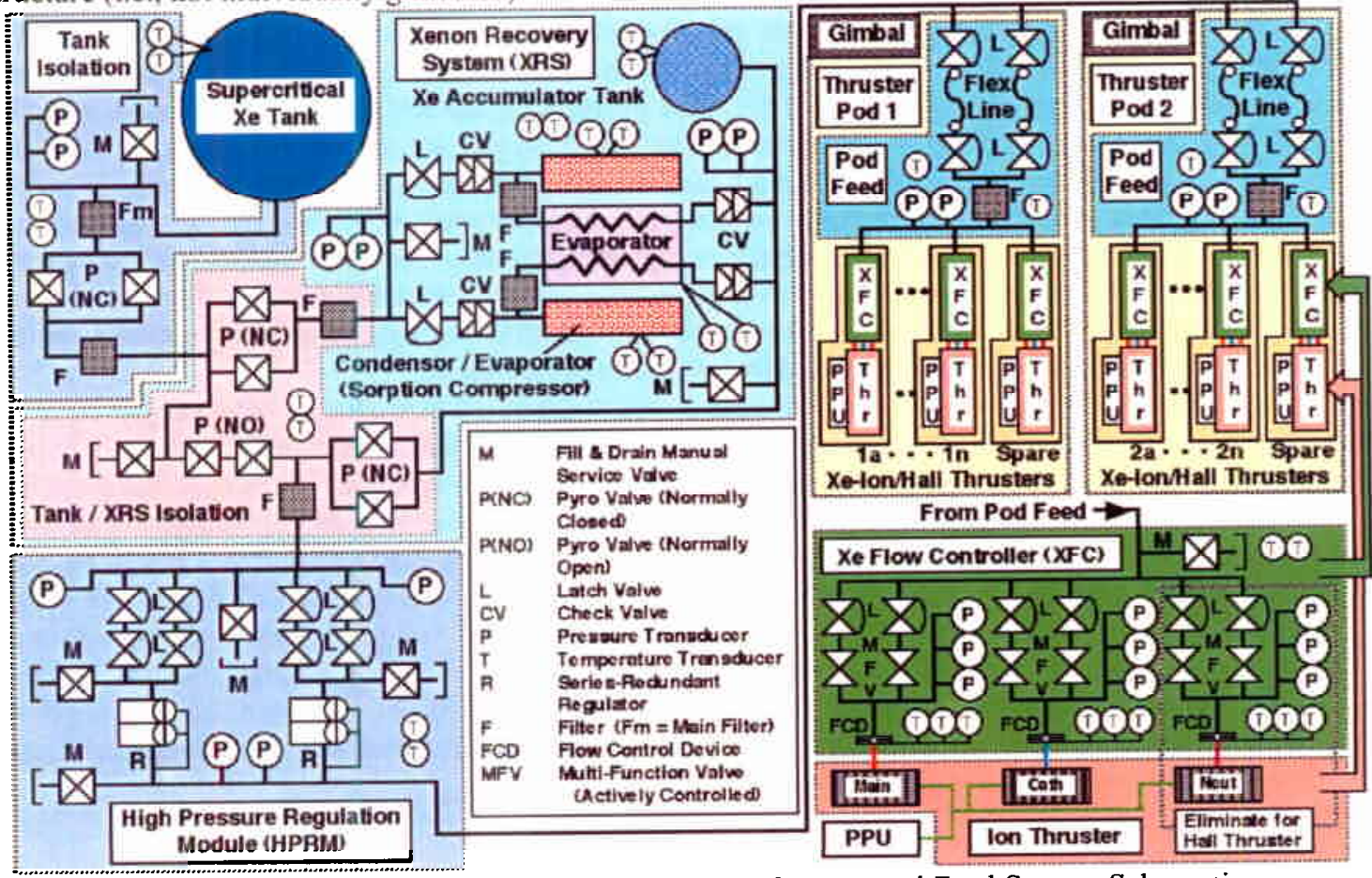

Figure 8. Xe-Propellant Ion (Herakles) Thruster Storage and Feed System Schematic.

Table 3. Summary of Propellant Storage and Feed System Mass and Power Scaling for the Xe-Ion (Herakles) Thruster System. (All masses in $\mathrm{kg}$ and powers in $\mathrm{W}_{\mathrm{e}}$.)

\begin{tabular}{|l|c|c|c|}
\hline Component & Fixed Mass, Power, or Parts Term & $\%$ of $\mathrm{M}_{\mathrm{p}}$ Term & $\%$ of $\left(\mathrm{M}_{\mathrm{p}}\right)^{23}$ Term \\
\hline Storage \& Feed System up to Pod Feed & 63.85 & \multirow{2}{*}{$2.5770 \%$} & $1.5232 \%$ \\
Mass & 6.3 & & $7.7371 \%$ \\
Power & 55 & \\
Parts Count & 13.90 & \\
Pod Feed System (I per Pod) & 0.0 & \\
Mass (Includes Gimbal) & 12 & \\
Power & 14.22 & \\
Parts Count & 0.0 & \\
Thruster Feed System (I per Thruster) & 26 & \\
Mass & & \\
Power & & \\
Parts Count & & \\
\hline
\end{tabular}




\section{Thruster Number Calculation Methodology}

For both the Ion and VHITAL propulsion systems, the number of thrusters (and their corresponding PPUs, feed systems, etc.) required in each pod will be a function of the total "bus" power and the power-per-thruster of each thruster. Specifically, we first calculate the net total power available to the thrusters by subtracting all the various system electric powers (e.g., systems, payload, RCS catalyst bed heaters, propellant storage and feed system heaters, etc.) from the total "bus" power. We then determine the power-per-PPU for the thrusters, which is equal to the thruster's power-per-thruster (typically a function of the thruster's $I_{s p}$ ) divided by the PPU's efficiency. The number of operating thrusters is then simply the rounded-up integer of (available power)/(power-per-PPU). For the Ion thruster system with two pods, it may be necessary to add one additional thruster so that each pod has the same number of operating engines so as to ensure overall thrust balance (e.g., left/right symmetry). Also, for thrusters with short lifetimes (i.e., low propellant throughput-per-thruster), it may be necessary to add extra complete sets of thrusters so as to consume all of the propellant for the mission. Finally, one extra thruster (and its PPU) is added to each pod as a redundancy spare. As a specific numerical example, if we have a $6,000-1 b_{r} s / b_{m} I_{s p}$ lon propulsion system for a $1,000-\mathrm{kW}_{\mathrm{e}}$ total "bus" power vehicle, the power available to the thrusters is $998.2 \mathrm{~kW}_{\mathrm{e}}$. The power-perPPU is $23.9 \mathrm{~kW}_{\mathrm{e}}$, so there are $(998.2 / 23.9)=41.7$ operating, which is rounded up to 42 thrusters. (This corresponds to each of the 42 thrusters operating at $99.3 \%$ of their full rated power.) For the Ion system, we have two pods, which implies a need for an even total number of operating thrusters. In this case, we already have an even total number (i.e., 42 total, with 21 in each pod), so we do not need to add an additional thruster for thrust balance. (If there were only one pod, then an even or odd number of operating thrusters would be allowed based on the assumption that any gimbaling required to compensate for thrust imbalance would be small, and thus would not impact overall performance, because all the thrusters would be relatively near the vehicle's thrust centerline. By contrast, thrusters in separate pods would be far from the vehicle thrust centerline, and thus produce a thrust imbalance moment-arm that could not be corrected by gambling without unduly impacting performance.)

The lon thrusters have a long lifetime, so only one set of operating thrusters is required to consume all of the Xe propellant. Otherwise, it would be necessary to add additional sets of 42 thrusters (again distributed evenly between the pods) until a cumulative total throughput (lifetime) was reached that consumed all of the required propellant. In this case, the number of sets (with 42 thrusters in each set) would be the rounded-up integer value of (total propellant mass)/(total throughput of 42 thrusters). Again, one extra spare thruster (and PPU) would be added to each pod for redundancy. Finally, when we consider $\mathrm{MW}_{\mathrm{e}}$-class electric propulsion vehicles, systems using thrusters with modest power-per-thruster and modest lifetimes (throughput) will only require one set of thrusters because the large total number of thrusters required to consume the available $M W_{e}$ of power naturally results in sufficient numbers of thrusters to consume the available amount of propellant. Typically, the need for additional sets of thrusters arises only when we have the combination of high power-per-thruster and low throughput-per-thruster, as can be the case with magnetoplasmadynamic (MPD) thrusters.

\section{VHITAL Propulsion System}

The VHITAL thruster was described in detail in a previous section. For these analyses, we have assumed a thruster mass of $36 \mathrm{~kg}$. The VHITAL nominal (baseline) $I_{\mathrm{sp}}$ assumed for these analyses is $6,000 \mathrm{lb}_{\mathrm{r}}-\mathrm{s} / \mathrm{lb}_{\mathrm{m}}$, with a power-per-thruster of $25.00 \mathrm{~kW}_{\mathrm{e}}$ and an efficiency of $76.1 \%$ at $6,000 \mathrm{lb}_{\mathrm{r}} \mathrm{s} / \mathrm{lb}_{\mathrm{m}} \mathrm{I}_{\mathrm{sp}}$. The assumed VHITAL propellant throughput is $2,150 \mathrm{~kg}$ (independent of $\mathrm{l}_{\mathrm{sp}}$ ). We also evaluated the VHITAL operating at $\mathrm{l}_{\mathrm{sp}}$ values ranging from 5,000 to $8,000 \mathrm{Ib}_{\mathrm{r}}-\mathrm{s} / \mathrm{lb}_{\mathrm{m}}$, with power-per-thruster and efficiency ranging from $18.75 \mathrm{~kW}_{\mathrm{e}}$ and $75.7 \%$, to $36.00 \mathrm{~kW}_{\mathrm{e}}$ and $76.8 \%$, respectively, as shown in Figure 9 . 


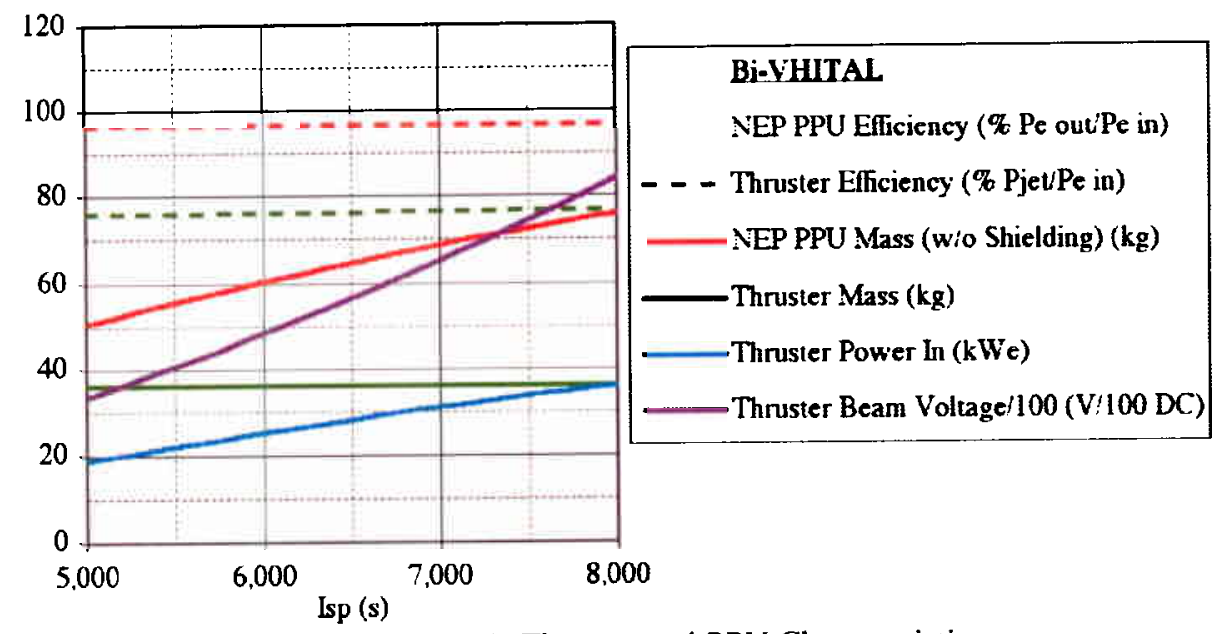

Figure 9. VHITAL Thruster and PPU Characteristics.

However, the thruster mass quoted above does not include a power processing unit (PPU) that converts the NEP "bus" electric power (e.g., high-voltage AC) to the high-voltage DC power required by the VHITAL thruster. Based on mass and efficiency scaling models for Ion thruster PPUs, we have estimated the mass of a VHITAL NEP PPU as $60.7 \mathrm{~kg}$ with an efficiency of $96.5 \%$ at an Isp of $6,000 \mathrm{lb}-\mathrm{s} / \mathrm{lb}_{\mathrm{m}}$. The variation in PPU mass and efficiency as a function of thruster power (i.e., $\mathrm{I}_{\mathrm{sp}}$ ) is shown in Figure 10.

Also, a mass list for a bismuth propellant storage and feed system was developed based on a design by Joseph Lewis (JPL). For the Bi-propellant VHITAL, we can store the propellant before launch as a room-temperature solid in the propellant tank, and then melt the Bi once in space. (This step can occur at an arbitrarily slow rate so as to not represent a major electric power impact, even though the full energy [enthalpy] of melting would need to be provided.) For calculation purposes, the thermal control (heating) power requirement for maintaining the propellant tank and feed system was determined by Robert Miyake (JPL) based on an assumed storage temperature of $280^{\circ} \mathrm{C}$. (Bi melts at $271^{\circ} \mathrm{C}$.) Also, as with the Ion thruster, we have assumed the use of two thruster pods because of the modest power-per-thruster of the VHITAL engine.

A schematic of the Bi-VHITAL propellant storage and feed system is shown in Figure 10. A summary of the various components is given in Table 4 . In this system, a low-pressure (few psi) sequential-blowdown helium pressurization system is used to pressurize the molten $\mathrm{Bi}$; this ensures that any $\mathrm{Bi}$ vapors that could diffuse into the pressurization system cannot cause a contamination-induced failure because the vapors are allowed to "see" only already-discharged He pressurization modules (and are isolated from un-used units by normally-closed pyro valves). With sufficient head pressure, the liquid metal is then forced into the electromagnetic pumps, which are used to push the liquid through the feed system and into the thrusters where the liquid is vaporized just prior to entry into the VHITAL engine. Interestingly, the electromagnetic pumps serve the same general purpose as pressure regulators and valves in the Xe system; similarly, Bi flow sensors are analogous to gas pressure gages in a Xe system. Note that significant electric power is required to maintain the system at an elevated temperature, as well as additional electric power corresponding to the heat of vaporization of bismuth (i.e., the power is equal to the heat of vaporization $[501 \mathrm{~J} / \mathrm{g}]$ multiplied by the mass flow rate $[\mathrm{g} / \mathrm{s}]$ of propellant into the VHITAL thrusters).

Finally, it is important to emphasize the extraordinarily low tankage mass of the Bi propellant tankage due to bismuth's high density. For example, the $M_{p}$ mass term in Table 4 is only $0.509 \%$ as compared to $2.577 \%$ for supercritical Xe. As we will see below, this has a profound effect on overall vehicle performance because, as shown in Figure 11, the tankage dry mass is so low for Bi. Thus, the high density of Bi more than compensates for the added propellant storage and feed system component masses, heater powers, etc., thus pointing to the importance of dry mass as well as $I_{s p}$ in determining overall vehicle performance. Additionally, the VHITAL thruster has a slightly higher efficiency and power-per-thruster than the Ion system, providing even greater benefits. 

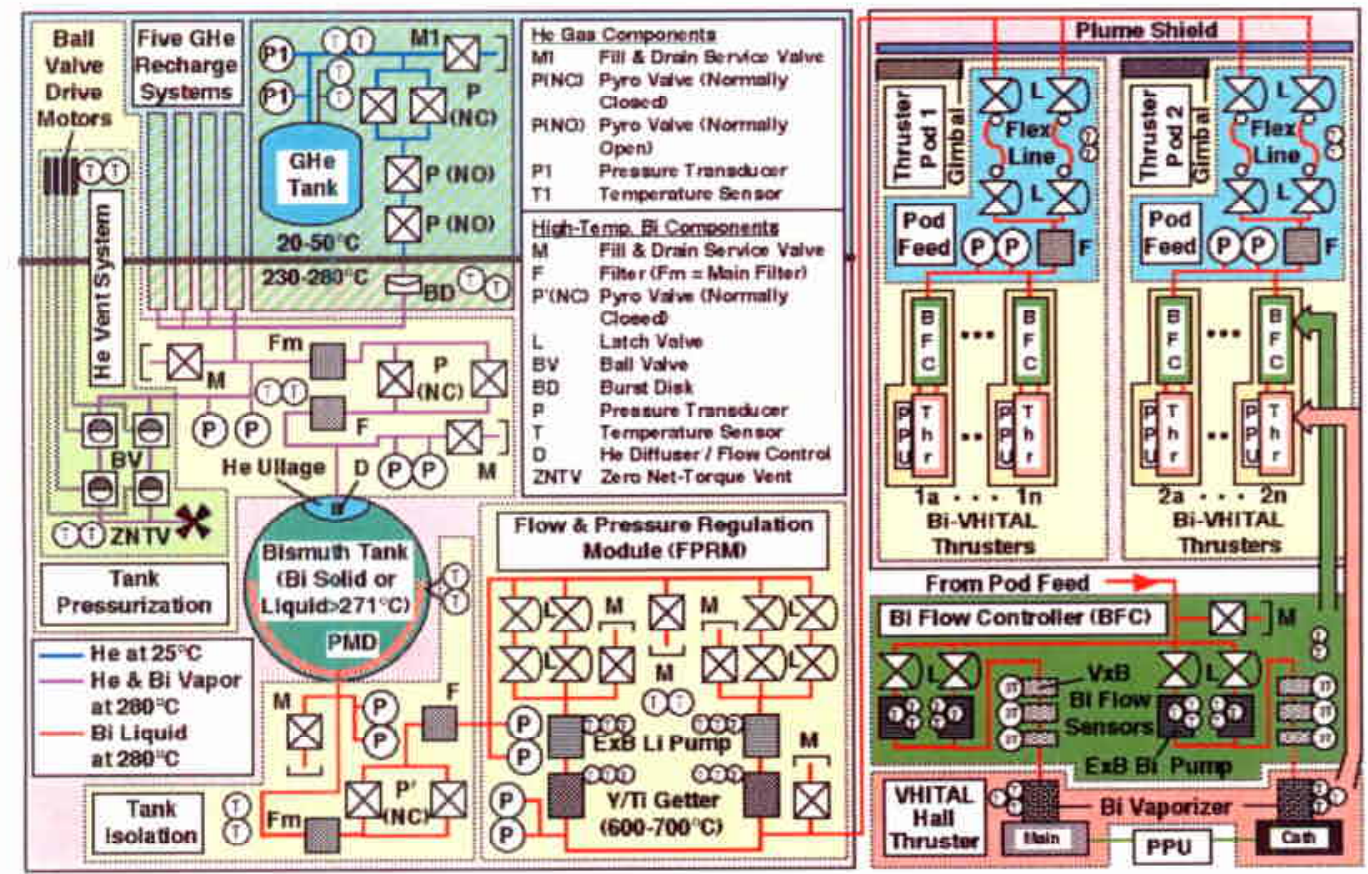

Figure 10. Bi-Propellant VHITAL Storage and Feed System Schematic.

Table 4. Summary of Propellant Storage and Feed System Mass and Power Scaling for the Bi-VHITAL System. (All masses in $\mathrm{kg}$ and powers in $\mathrm{W}_{\mathrm{e}}$.)

\begin{tabular}{|l|c|c|c|}
\hline Component & Fixed Mass, Power, or Parts Term & $\%$ of $\mathrm{M}_{\mathrm{p}}$ Term & $\%$ of $\left(\mathrm{M}_{\mathrm{p}}\right)^{2.3}$ Term \\
\hline Storage \& Feed System up to Pod Feed & 66.10 & $0.5094 \%$ & $1.0574 \%$ \\
Mass & 100.8 & & $31.8177 \%$ \\
Power & 98 & \\
Parts Count & 17.67 & \\
Pod Feed System (1 per pod) & 25.2 & \\
Mass (includes gimbal) & 12 & \\
Power & 21.9 & \\
Parts Count & 25.2 & & \\
Thruster Feed System (1 per thruster) & 16 & \\
Mass & & \\
Power & & \\
Parts count & & \\
\hline
\end{tabular}




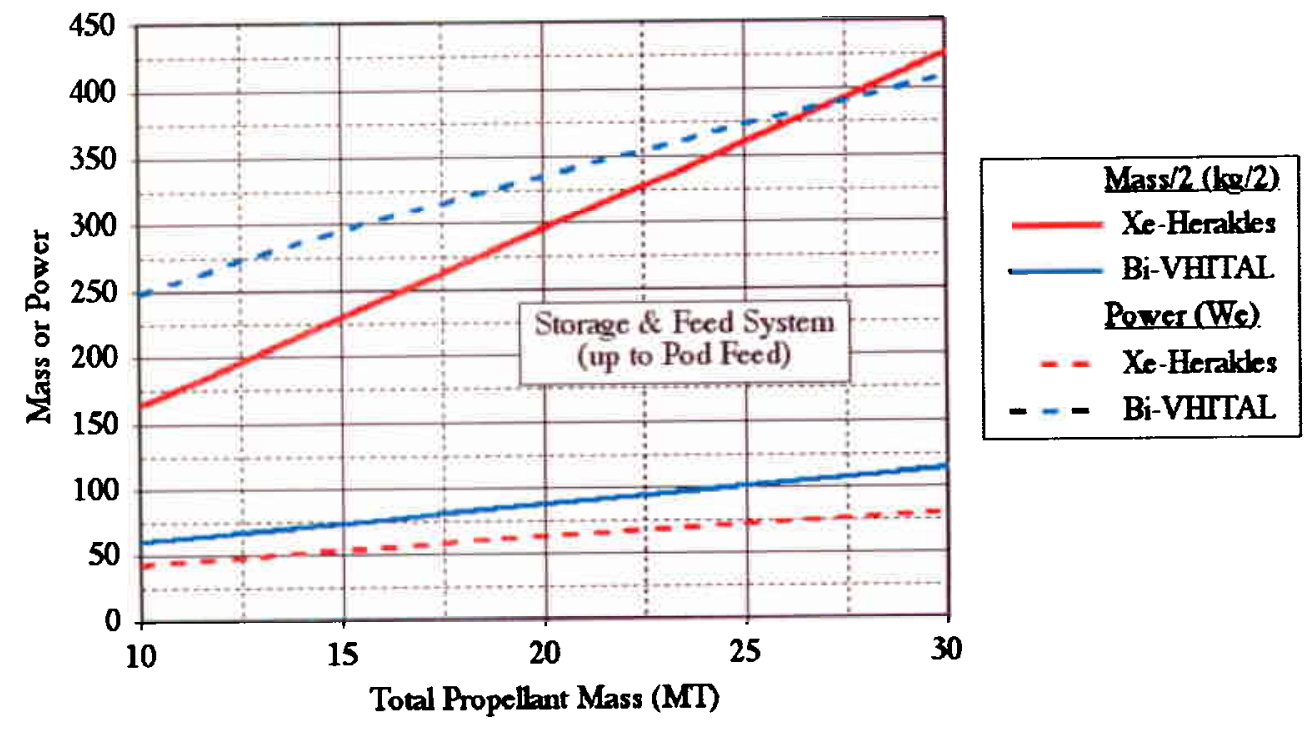

Figure 11. Comparison of Xe-Ion (Herakles) and Bi-VHITAL Storage and Feed System Dry Mass and Thermal Control Heater Power. (Propellant Storage and Feed System up to Pod Feed.)

\section{Mission Analysis Results for the NEP Robotic Planetary Mission}

We evaluated two robotic missions in these analyses. The first was a Saturn Orbiter Mission that includes an extensive tour of Saturn's moons. The second mission was an Interstellar Precursor Mission to $200 \mathrm{AU}$ with a Solar System escape velocity $\left(V_{\mathrm{inf}}\right)$ of either 5 or $10 \mathrm{AU} / \mathrm{year}$. For each of these missions, we considered two primary factors in evaluating the mission benefits of the VHITAL propulsion system:

1. Total Initial Mass in Low Earth Orbit (IMLEO) and Trip Time

2. Propulsion System Complexity (as represented by a parts count)

\section{Initial Mass in Low Earth Orbit (IMLEO) vs Trip Time for the Saturn Orbiter with Moon Tour}

Figure 12 illustrates the IMLEO versus trip time performance for the Ion (Herakles) and VHITAL systems over a range of $I_{s p}$ from 6,000 to $8,000 \mathrm{lb}_{\mathrm{t}}-\mathrm{s} / \mathrm{lb}_{\mathrm{m}}$. These analyses show the importance of a high $I_{\mathrm{sp}}$ for high- $\Delta \mathrm{V}$ missions; for example, there is a pronounced difference between the $6,000-1 b_{\mathrm{f}}-\mathrm{s} / \mathrm{lb}_{\mathrm{m}} I_{\mathrm{sp}}$ curves and the 7,000- or $8,000-\mathrm{lb}_{\mathrm{f}}-\mathrm{s} / \mathrm{b}_{\mathrm{m}} \mathrm{I}_{\mathrm{sp}}$ curves. This is primarily due this being a high $\Delta \mathrm{V}$ (ca. $41 \mathrm{~km} / \mathrm{s}$ total) mission. Nevertheless, at any given $I_{s p}$, the VHITAL system is superior to the lon system, with the slightly higher VHITAL thruster efficiency, power-per-thruster, and lower tankage mass of $\mathrm{Bi}$ propellant providing superior performance in terms of initial launch mass and trip time.

Also worth noting is the "jog" in the VHITAL curve at an $I_{s p}$ of $6,000 \mathrm{lb}_{r}-\mathrm{s} / / \mathrm{b}_{\mathrm{m}}$ below $300 \mathrm{~kW}_{\mathrm{e}}$. This is due to the limited lifetime (throughput) of the VHITAL thruster. For example, at the power level point of $300 \mathrm{~kW}_{\mathrm{e}}$ or higher, the number of thrusters required to consume the available power (e.g., 12 running VHITAL thrusters [plus 1 spare in each pod] at a total "bus" power of $300 \mathrm{~kW}_{\mathrm{e}}$ ) is sufficient to consume the total amount of propellant. However, at $250 \mathrm{~kW}_{\mathrm{e}}$, the 10 running VHITAL thrusters are insufficient to consume all of the propellant, so an extra set of 10 thrusters (along with their corresponding PPUs, feed systenis, gimbals, etc.) must be added so as to accommodate all the propellant, resulting in a total of 22 thrusters (including 1 spare in each pod). At higher values of $I_{\text {sp, }}$, the total propellant mass is sufficiently reduced so that additional sets of thrusters are not needed at power levels as low as $200 \mathrm{~kW}_{\mathrm{e}}$. 

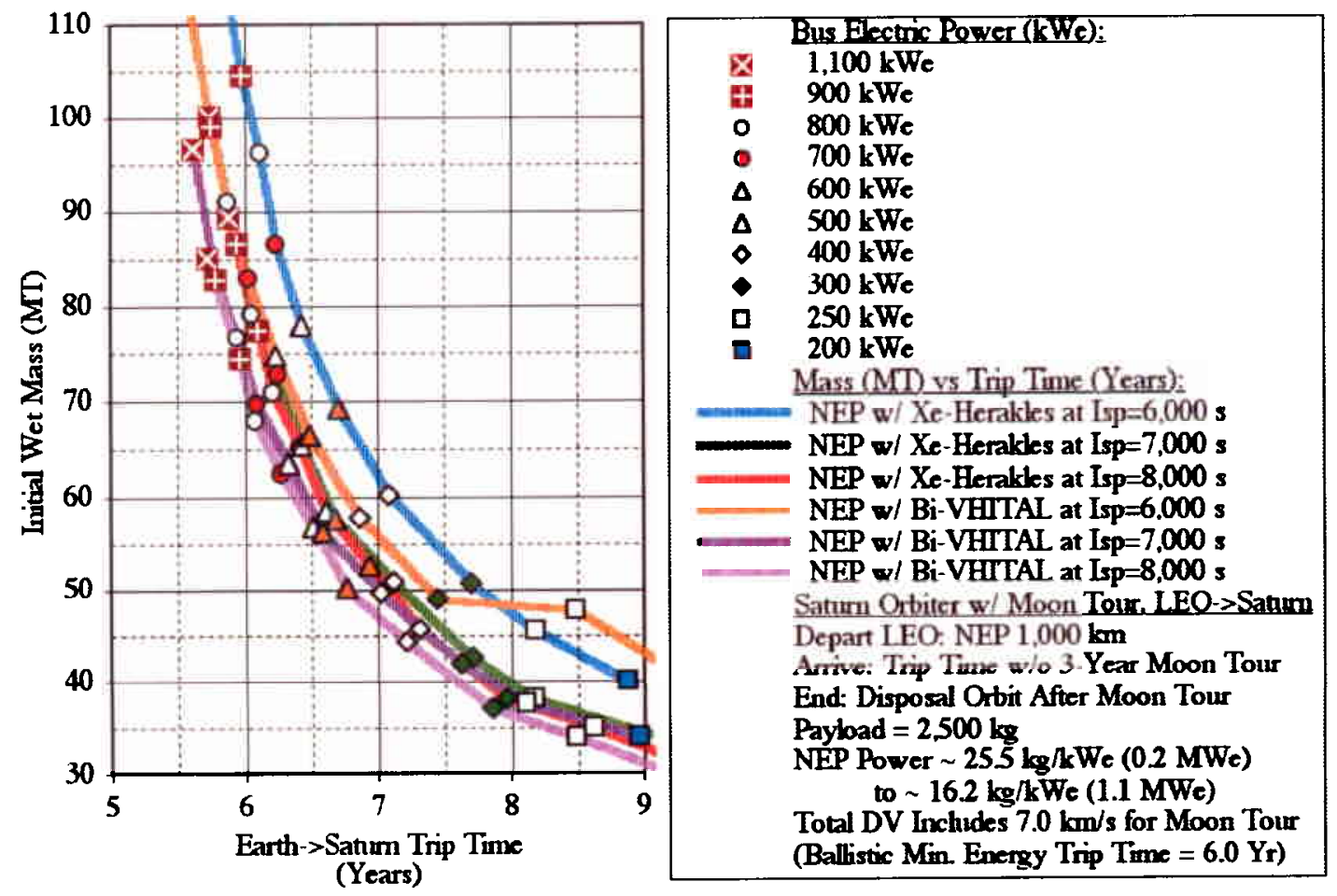

Figure 12. Variation in IMLEO and Trip Time for the Saturn Orbiter with Moon Tour Mission.

\section{Initial Mass in Low Earth Orbit (IMLEO) vs Trip Time for the Interstellar Precursor Mission}

Figure 13 illustrates the IMLEO versus trip time (to $200 \mathrm{AU}$ ) performance for the lon (Herakles) and VHITAL thruster types over a range of $I_{s p}$ from 6,000 to $8,000 \mathrm{lb}_{\mathrm{r}}-\mathrm{s} / \mathrm{lb}_{\mathrm{m}}$. Performance for two cases is given; the first is for a slower final velocity $\left(V_{\text {inf }}\right)$ of 5 Astronomical Units ( $\left.A U\right)$ per year, and the second is for a faster $V_{\text {inf }}$ of $10 \mathrm{AU} / \mathrm{year}$. Not surprisingly, the slower mission is less demanding in overall mass and required power. As with the Saturn Mission, these analyses show the importance of a high $I_{s p}$ for high- $\Delta V$ missions; for example, there is little difference between the higher $I_{\mathrm{sp}}$ values for the $5 \mathrm{AU} / \mathrm{Yr}$ mission which has a $\Delta V$ on the order of $35 \mathrm{~km} / \mathrm{s}$ (comparable to the Saturn Mission total $\Delta \mathrm{V}$ ); by contrast, there is a strong $1_{\text {sp }}$ dependence for the $10 \mathrm{AU} / \mathrm{year}$ mission with a $\Delta \mathrm{V}$ around $60 \mathrm{~km} / \mathrm{s}$.

As with the Saturn mission, we again see a "jog" in the VHITAL curve for the $10 \mathrm{AU} / \mathrm{Y}$ ear mission at an $I_{\text {sp }}$ of $8,000 \mathrm{lb}_{\mathrm{r}}-\mathrm{s} / \mathrm{lb}_{\mathrm{m}}$ between the 1,400 and $1,500 \mathrm{~kW}_{\mathrm{e}}$ points due to the limited lifetime (throughput) of the VHITAL thruster. In this case, at a power level of $1,500 \mathrm{~kW}_{\mathrm{e}}$ or more, the number of thrusters required to consume the available power is sufficient to consume the total amount of propellant whereas at $1,400 \mathrm{~kW}_{e}$, an extra set of thrusters must be added so as to accommodate all the propellant. Similarly, at an $I_{s p}$ of 6,000 and $7,000 \mathrm{lb}_{\mathrm{r}}-\mathrm{s} / \mathrm{lb}_{\mathrm{wn}}$, the total propellant load is large enough that two sets of VHITAL thrusters are needed throughout the range of powers.

Again, as with the Saturn Mission, we see that the VHITAL system is superior to the lon system for the lower $\Delta V$ case (i.e., $35 \mathrm{~km} / \mathrm{s}$ at $5 \mathrm{AU} /$ year). However, for the very high- $\Delta \mathrm{V} 10 \mathrm{AU} /$ year mission with its higher propellant load, the higher throughput of the lon thruster results in a smaller overall number of thrusters (and ultimately dry mass), resulting in generally superior performance. Note that there can be a complex interaction between the various factors ( $I_{\text {sp }}$ and $\Delta V$, power per thruster and throughput per thruster, and so on) that is very mission dependent. Thus, for the more modest $\Delta \mathrm{V}$ Saturn and $5 \mathrm{AU} /$ year $200 \mathrm{AU}$ missions, the total propellant masses and total "bus" powers are low; for the cases at low power levels where there are insufficient VHITAL thrusters to consume all the propellant, adding an extra set of thrusters is not a major mass impact simply because the total number of running thrusters is small. By contrast, in the high- $\Delta \mathrm{V}$ and high-power $10 \mathrm{AU} /$ year $200 \mathrm{AU}$ mission, the throughput difference is especially detrimental because of both the very high propellant load and the very large number of thrusters required at high power; here, having to add an extra set of thrusters has a significant mass impact because of the large number of thrusters running at high powers. This issue of number of thrusters, and its impact on overall system complexity, is discussed in more detail below. 

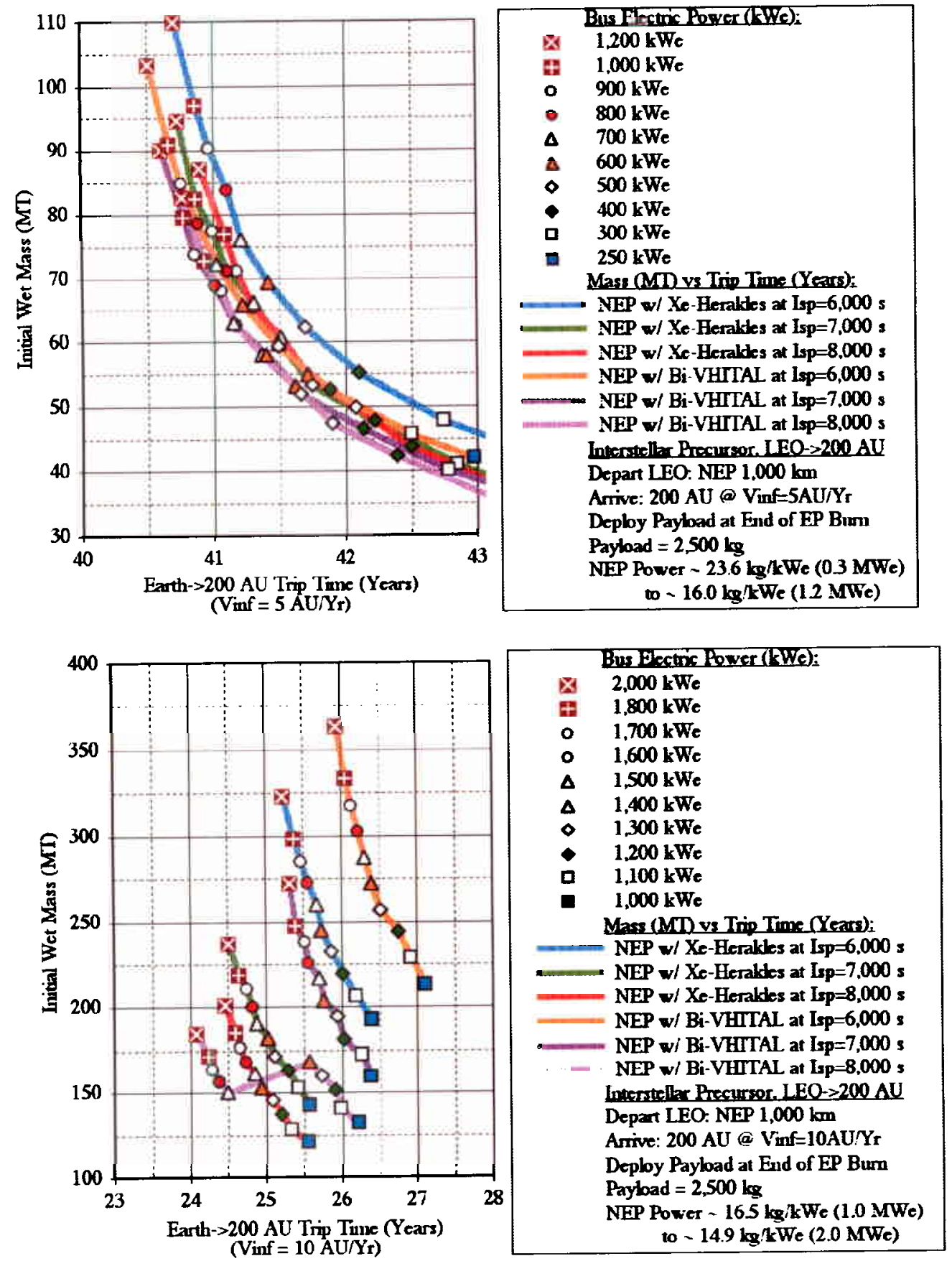

Figure 13. Variation in IMLEO and Trip Time for the $200 \mathrm{AU}$ Interstellar Precursor Mission. (Upper Figure $V_{\text {inf }}=5 \mathrm{AU} /$ Year; lower Figure $\mathrm{V}_{\text {inf }}=10 \mathrm{AU} / \mathrm{Year}$.)

\section{Propulsion System Complexity}

Thus far we have concentrated on mass and trip time as the traditional figures of merit in determining the benefit (i.e., feasibility) of the new propulsion technologies embodied in the VHITAL system. However, another element of mission feasibility is the overall system "complexity." Like beauty, "complexity" can be in the eye of the beholder and consist of many, often competing variables. Nevertheless, no matter how it is quantified, high "complexity" is generally considered undesirable because of its perceived impact on system reliability. 
Figure 14 illustrates the propulsion system parts count (e.g., thrusters, valves, regulators, filters, etc.) as a function of the total or "bus" power level for both the Saturn mission and the $10 \mathrm{AU} / \mathrm{year} 200 \mathrm{AU}$ mission. In this case, the parts count is used as a measure of system "complexity." For example, when extra sets of thrusters are not required for throughput considerations, the VHITAL propulsion system, with its somewhat higher power-perthruster, has a smaller parts count, and thus ultimately "complexity," for components like the number of thrusters, valves, etc. By contrast, adding extra sets of thrusters for adequate total throughput can dramatically increase the parts count for the VHITAL system, especially for a high-power system with many running thrusters.
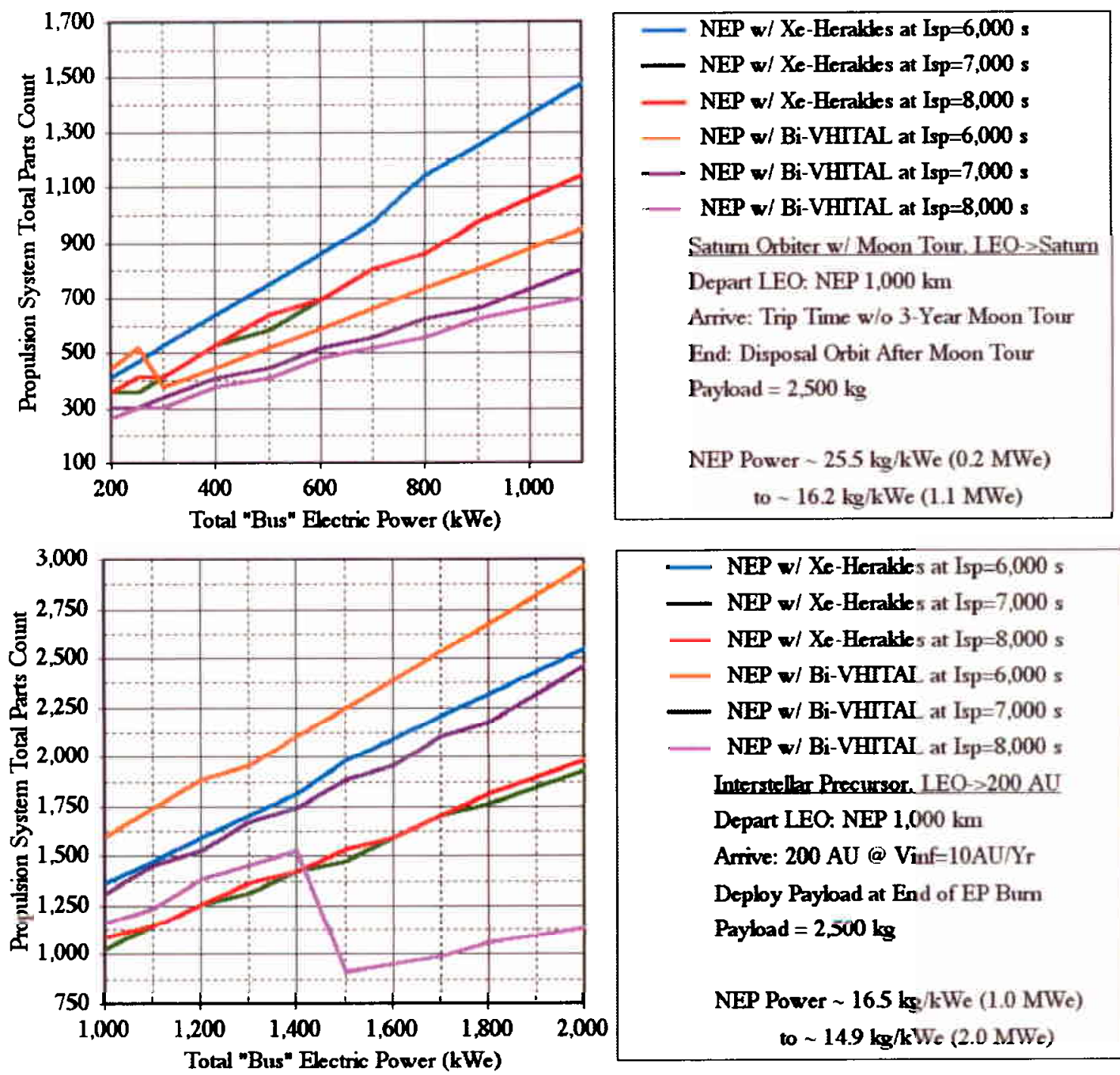

\section{NEP $w / X_{e}$-Herakdes at Isp $=6,000 s$ \\ - NEP $w / X_{c}-$ Heraldes at Isp $=7,000 \mathrm{~s}$ \\ - NEP $w / X_{c}$-Heraldes at Isp $=8,000$ s \\ - NEP w/ Bi-VHITAL at Isp $=6,000$ s \\ - NEP $w /$ Bi-VHTTAL at Isp $=7,000 \mathrm{~s}$ \\ - NEP w/ Bi-VHITAL at Isp $=8,000 \mathrm{~s}$ \\ Interstellar Precirsor. LEO $\rightarrow 200$ AU \\ Depart LEO: NEP $1,000 \mathrm{~km}$}

Arrive: $200 \mathrm{AU} @ \mathrm{Vinf}=10 \mathrm{AU} / \mathrm{Yr}$

Deploy Payload at End of EP Burn

Payload $=2.500 \mathrm{~kg}$

NEP Power $\sim 16.5 \mathrm{~kg} / \mathrm{kWe}(1.0 \mathrm{MWe})$

to $\sim 14.9 \mathrm{~kg} / \mathrm{kWe}(2.0 \mathrm{MWc})$

Figure 14. Electric Propulsion System Parts Count vs Total "Bus" Electric Power for the NEP Saturn Orbiter with Moon Tour Mission (Upper Figure) and $200 \mathrm{AU}$ Interstellar Precursor Mission with $V_{\mathrm{inf}}=5 \mathrm{AU} /$ Year (Lower Figure).

Also, based on theses analyses, we can determine the increase in throughput needed for the VHITAL thruster that would be required to eliminate the need for additional thrusters. For example, if we wanted to have a single set of VHITAL thrusters at even the lowest power levels for the high- $\Delta \mathrm{V} 10 \mathrm{AU} / \mathrm{year} 200 \mathrm{AU}$ mission, it would be necessary to increase the baseline throughput $(2,150 \mathrm{~kg})$ by only $42 \%$ for the $6,000-\mathrm{lb}_{\mathrm{f}}-\mathrm{s} / \mathrm{lb}_{\mathrm{m}} \mathrm{I}_{\mathrm{sp}}$ system, $24 \%$ for the $7,000-1 b_{\mathrm{f}}-\mathrm{s} / \mathrm{lb}_{\mathrm{m}}$ system, and $9 \%$ for the $8,000-\mathrm{lb}_{\mathrm{r}}-\mathrm{s} / \mathrm{b}_{\mathrm{m}}$ systems. This suggests that a program to increase the VHITAL thruster's throughput by even modest amounts over the assumed baseline value could have significant mission benefits, as shown in Figures 15 (IMLEO vs trip time) and 16 (parts count vs power level). 


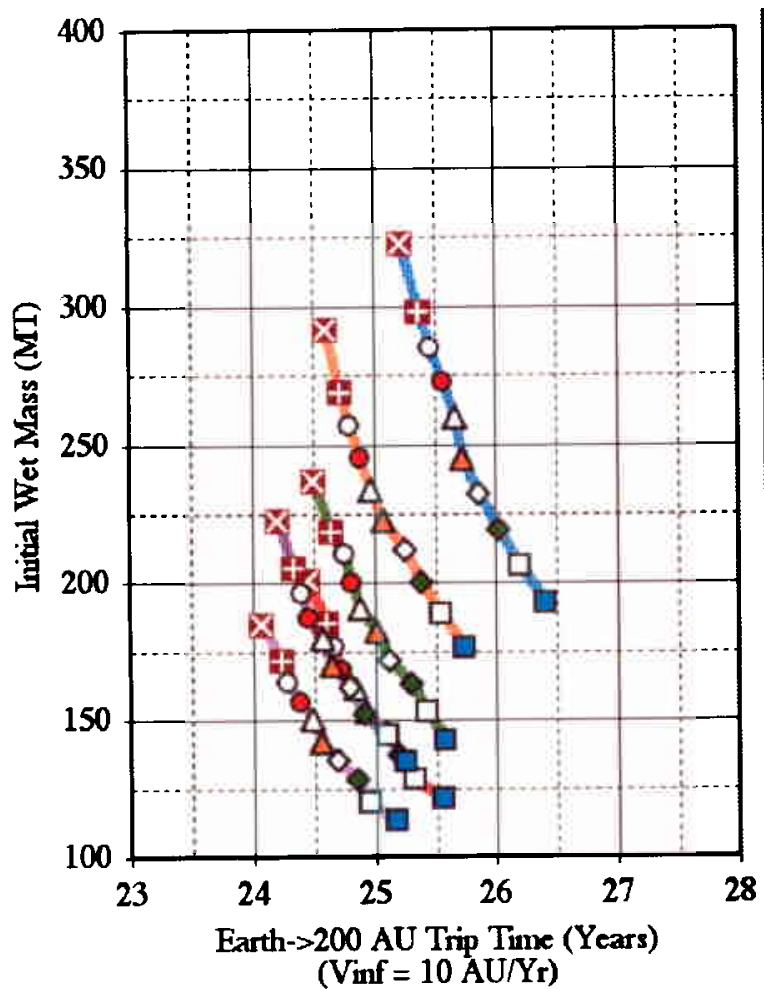

\begin{tabular}{|c|c|}
\hline & Bus Flectric Power (kWe): \\
\hline$x$ & $2,000 \mathrm{kWe}$ \\
\hline F & $1,800 \mathrm{kWe}$ \\
\hline $\mathbf{0}$ & $1,700 \mathrm{kWe}$ \\
\hline D & $1,600 \mathrm{kWe}$ \\
\hline$\Delta$ & $1,500 \mathrm{kWe}$ \\
\hline$\Delta$ & $1,400 \mathrm{kWe}$ \\
\hline$\bullet$ & $1,300 \mathrm{kWe}$ \\
\hline$\bullet$ & $1,200 \mathrm{kWe}$ \\
\hline $\mathbf{\square}$ & $1,100 \mathrm{kWe}$ \\
\hline & $1,000 \mathrm{kWe}$ \\
\hline & Mass (MD) ys Trip Time (Years): \\
\hline 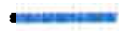 & NEP $w / X_{e}-H_{c r a l d e s}$ at Isp $=6,000 \mathrm{~s}$ \\
\hline & $\begin{array}{l}\text { NEP } w / \text { Xe-Heraldes at Isp }=7,000 \mathrm{~s} \\
\text { NEP } w / \text { Xe-Heraldes at Isp=8,000 s }\end{array}$ \\
\hline$=$ & NEP $w /$ Bi-VHITAL at Isp $=6,000 \mathrm{~s}$ \\
\hline vendenobuve & NEP $w / B i-V H I T A L ~ a t ~ I s p=7,000 s$ \\
\hline mann & NEP $w / B i-V H T T A L$ at Isp $=8,000 \mathrm{~s}$ \\
\hline & Interstellar Precursor. LEO $>200 \mathrm{AU}$ \\
\hline & Depart LEO: NEP 1,000 km \\
\hline & Arrive: $200 \mathrm{AU} @$ Vinf=10AU/Yr \\
\hline & Payload = $2,500 \mathrm{~kg}$ \\
\hline & $\begin{array}{c}\text { NEP Power }-16.5 \mathrm{~kg} / \mathrm{kWe}(1.0 \mathrm{MWe}) \\
\text { to } \sim 14.9 \mathrm{~kg} / \mathrm{kWe}(2.0 \mathrm{MWe})\end{array}$ \\
\hline
\end{tabular}

Figure 15. Variation in IMLEO and Trip Time for the $200 \mathrm{AU}$ Interstellar Precursor Mission with $\mathrm{V}_{\text {inf }}=5 \mathrm{AU} / \mathrm{Year}$ and VHITAL Throughput Adjusted to Eliminate Need for Additional Thruster Sets.
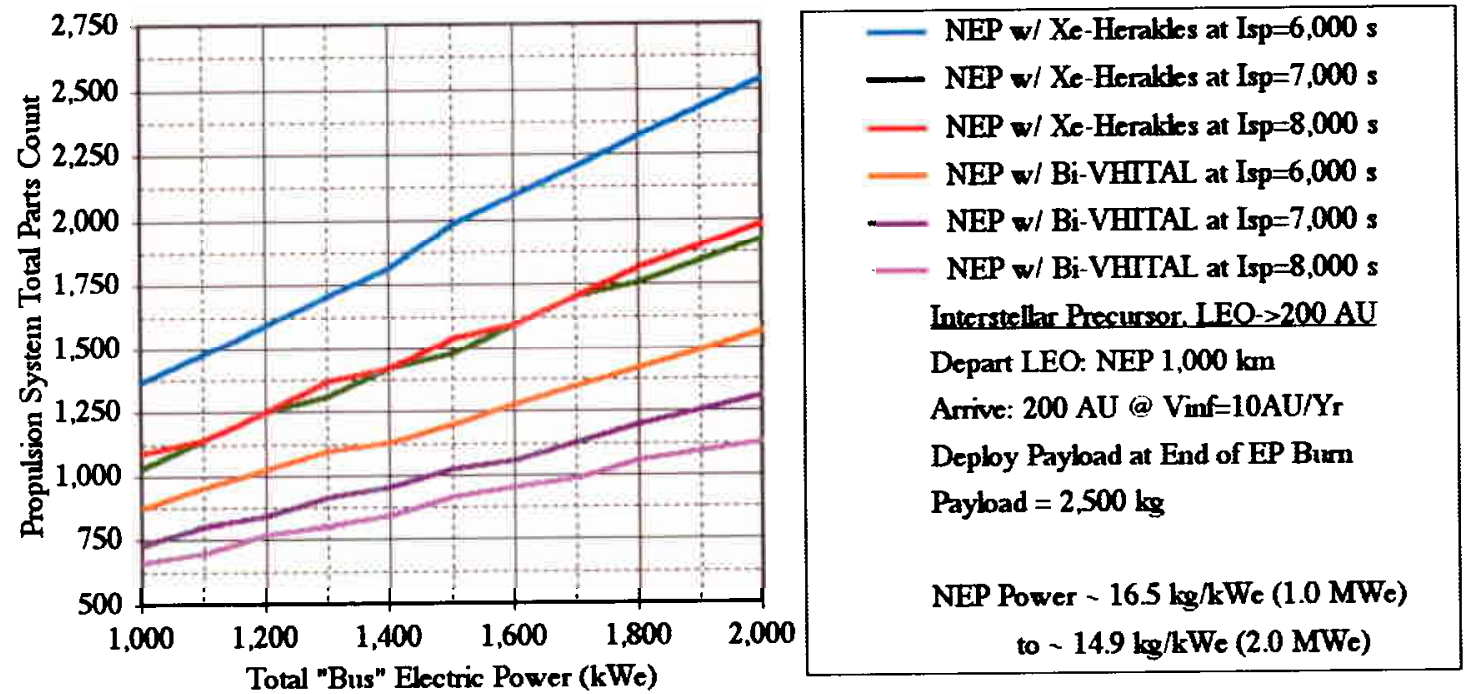

Figure 16. Electric Propulsion System Parts Count vs Total "Bus" Electric Power for the NEP 200 AU Interstellar Precursor Mission with $V_{\text {inf }}=5$ AU/Year and VHITAL Throughput Adjusted to Eliminate Need for Additional Thruster Sets.

Finally, although not explicitly considered in detail in this study, there is the non-trivial issue of packaging a large number of thrusters in the Earth-launch vehicle launch shroud. For example, the Herakles lon thruster has roughly 2.9 the diameter of the VHITAL (including mounting bracket); even though there are 1.17 VHITAL for 
every one Ion thruster due to the difference in power-per-thruster, we find that there is a significant packaging and integration challenge for Ion thrusters in MW-class electric propulsion systems, as illustrated in the Figure below.

Ion (Herakles): $16+1$ (Spare) per Pod, 2 Pods

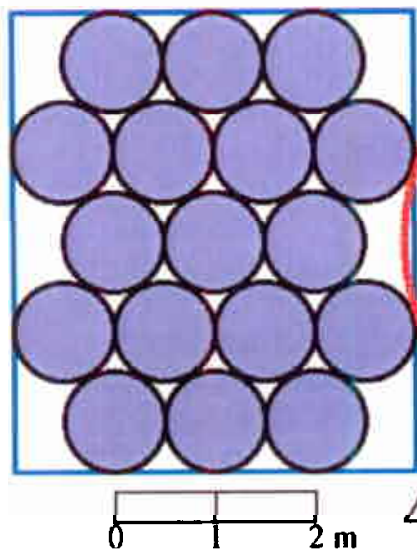

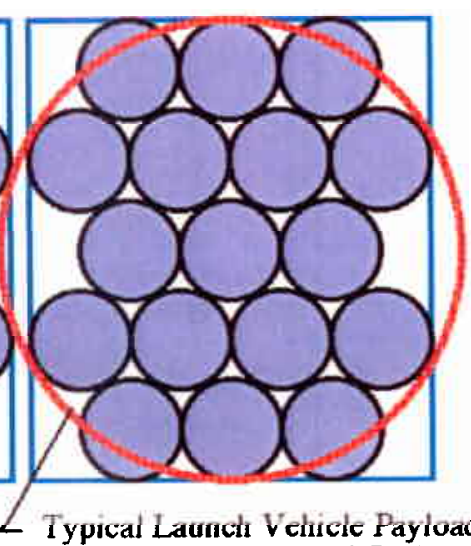

Diameter $(4.5 \mathrm{~m})$
VHITAL: $19+1$ (Spare) per Pod, 2 Pods

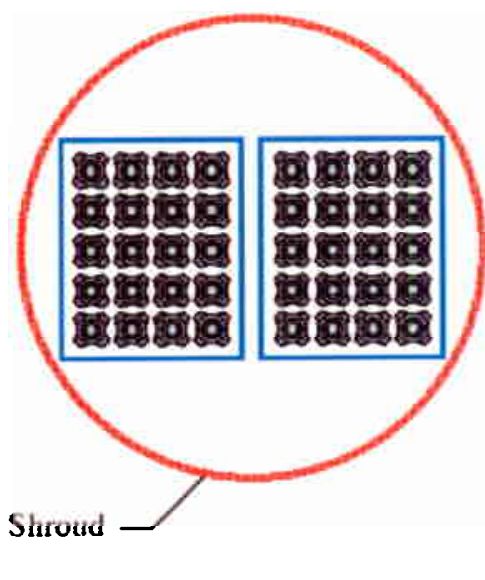

Lon: $31.2 \mathrm{kWe}$ into NEP PPU (Isp $=7,000 \mathrm{~s})$
Total("Bus") Power: I MWe
VHITAL: $26.6 \mathrm{kWe}$ into NEP PPU $($ Isp $=6,000 \mathrm{~s})$

Figure 17. Size Comparison Between Ion (Herakles) and VHITAL Thrusters for a 1-MW. Total "Bus" Power.

\section{Summary of Mission Analysis Results of VHITAL for NEP Robotic Planetary Missions}

As shown above, these high- $\Delta \mathrm{V}$ outer Solar System missions, where the vehicle spends most of its flight time in heliocentric space, tend to optimize towards high values of $I_{\mathrm{sp}}$, with an optimum in the 7,000 to $8,000 \mathrm{lb}_{\mathrm{f}}-\mathrm{s} / \mathrm{lb}_{\mathrm{m}} \mathrm{l}_{\mathrm{sp}}$ range. This may represent a significant challenge for the Bi-VHITAL system; however, as will be shown below, missions within the inner Solar System tend to favor lower $I_{\text {sp }}$ values, because a greater fraction of the time is spent in planetary gravity wells. In these cases, the lower $I_{\mathrm{sp}}$ produces more thrust (at a given power level), so as to potentially reduce the trip time. Nevertheless, even if limited to an $I_{\mathrm{sp}}$ of $7,000 \mathrm{lb}_{\mathrm{r}}-\mathrm{s} / \mathrm{l}_{\mathrm{m}}$, the Bi-VHITAL system is still competitive with a high-performance lon thruster system, with the very significant advantages of easier spacecraft integration and packaging within the launch vehicle (i.e., dramatically fewer thrusters), and especially reduced system complexity as evidenced by a roughly $30-40 \%$ reduction in the number of system components for the VHITAL system as compared to the Ion system.

\section{B. MISSION ANALYSIS RESULTS FOR THE NEP MARS CARGO MISSION}

For the Mars Cargo Mission, we have assumed the use of a reusable megawatt-class NEP vehicle for transport of payload from LEO to a $6,000-\mathrm{km}$ altitude low Mars orbit (LMO). Typical $\Delta V \mathrm{~V}$ for low-T/W LEO-toLMO transfers are on the order of $16 \mathrm{~km} / \mathrm{s}$. This LMO is at the same altitude as the inner, and larger moon of Mars, Phobos. This high-altitude LMO, rather than a low-altitude (e.g., $400-\mathrm{km}$ altitude) LMO was chosen to make it possible to support exploration of Phobos, with special emphasis on Phobos as a potential extraterrestrial resource of water that could be processed to produce chemical $\left(\mathrm{O}_{2} / \mathrm{H}_{2}\right)$ propellants. For example, after delivery of the cargo payloads, the NEP vehicle could land on Phobos and use its power to support mining, ore processing, water electrolysis, and so on. (Note that the chemical RCS thrusters might be needed for the landing, however, because the vehicle acceleration from the electric thrusters might be too small even for the micro-gravity surface gravity of Phobos.) Finally, the mass of Phobos could provide shielding mass to prevent radiation from the NEP vehicle from damaging other assets in Mars space.

Also, we have chosen an Earth-to-Mars trip time goal of 2.2 years to match the Earth-Mars synodic period. This makes it possible to launch the Cargo Vehicles during one trans-Mars injection (TMI) opportunity, travel to Mars, perform Mars orbit insertion (MOI), and check out all the payload systems prior to launching the crew during the next Mars TMl opportunity. 


\section{NEP System}

The assumptions made for the NEP vehicles described in a previous section are again used in this section.

\section{Mars Cargo Payload}

As with the human missions to the Moon, architectures for human exploration of Mars are still under study. For our EP Cargo Vehicle analyses, we have assumed a 63.892-MT payload derived from the NASA Human Exploration of Mars Design Reference Mission (DRM) Version 3.0. This payload corresponds to delivery of an Earth Return Vehicle (ERV) into Mars orbit. In the nominal DRM 3.0 Mission scenario, a Nuclear Thermal Propulsion (NTP) stage is used for Earth escape and trans-Mars injection (TMI); the NTP stage is then jettisoned, and the total payload (74.072 MT for the ERV and entry Aeroshell) is aerocaptured for Mars orbit insertion (MOI). For the electric propulsion options, the Aeroshell (10.180 MT) required for the nominal NTP ERV Cargo Mission is removed, because the EP Cargo Vehicle places the ERV directly into Mars orbit. There is also a second NTP Cargo Vehicle launch with a payload consisting of a 66.043-MT Cargo Lander (CL) (to place an Ascent Vehicle and other elements on the surface) that is aerobraked directly to the martian surface. Finally, the Crew Vehicle (with landers) is sent by NTP on a fast trajectory to Mars where the Crew Vehicle aerocaptures into Mars orbit.

Interestingly, if a slow, minimum-energy (Hohmann) trajectory is acceptable for the crew, an aerobraked chemical $\left(\mathrm{O}_{2} / \mathrm{H}_{2}\right)$ propulsion Crew Vehicle can provide comparable IMLEO to the NTP Crew Vehicle. However, the real advantage of NTP is its combination of high-T/W and high- $I_{\mathrm{sp}}$ (projected to be in the range of $940-960 \mathrm{lb}_{r}$ $\left.\mathrm{s} / \mathrm{l}_{\mathrm{m}}\right)$; this makes it possible to fly fast, high-energy trajectories that have much shorter flight times than that for the minimum-energy trajectory (e.g., 130-180 days versus the ideal 259 days, respectively, for the Earth-to-Mars step) without suffering from an excessive IMLEO.

\section{Mission Analysis Results for the NEP and Mars Cargo Mission}

As with the Lunar Cargo Mission, we considered the following factors:

1. Total Initial Mass in Low Earth Orbit (IMLEO) and Trip Time

2. Propulsion System Complexity (as represented by a parts count)

3. Vehicle Power Level Required for a Given Earth-to-Mars Trip Time (nominally 2.2 year)

\section{Initial Mass in Low Earth Orbit (IMLEO) vs Earth-to-Mars Trip Time}

As with the NEP robotic missions, we again see the similarity in performance between the VHITAL and Ion thruster systems for an NEP Cargo Mission, as shown in Figure 18. For this mission, with a $\Delta V$ typically on the order of $16 \mathrm{~km} / \mathrm{s}$, the optimum $l_{\mathrm{sp}}$ is around $6000-7,000 \mathrm{lb}_{\mathrm{f}}-\mathrm{s} / \mathrm{b}_{\mathrm{m}}$; higher $\mathrm{I}_{\mathrm{sp}}$ values result in the need for a higher power to achieve a given trip time such that the increase in power system mass (and corresponding increase in thruster, PPU, etc. mass) essentially negates any propellant (and propellant tankage) mass savings afforded by the higher $I_{\text {sp. }}$. Also, it is interesting to note that in the VHITAL NEP system, the optimum $I_{s p}$ is around $6,000-7,000 \mathrm{lb}_{r}$ $\mathrm{s} / \mathrm{b}_{\mathrm{m}}$ rather than the clearly optimum $7,000 \mathrm{lb}_{\mathrm{r}} \mathrm{s} / \mathrm{lb}_{\mathrm{m}}$ of the lon system; this effect is probably due to the interaction of the thruster efficiency, propulsion tankage dry mass, and acceleration ( $T / \mathrm{W}$ ratio). More specifically, vehicle thrust (and thus trip time) is proportional to the propulsion system's exhaust or "jet' power divided by $\mathbf{l}_{\mathrm{sp}}$; thus, the VHITAL, with its lower tankage mass, can more easily accommodate a higher propellant load from a lower $I_{\mathrm{sp}}$ in order to have the same vehicle acceleration as at a higher $I_{\mathrm{sp}}$. 

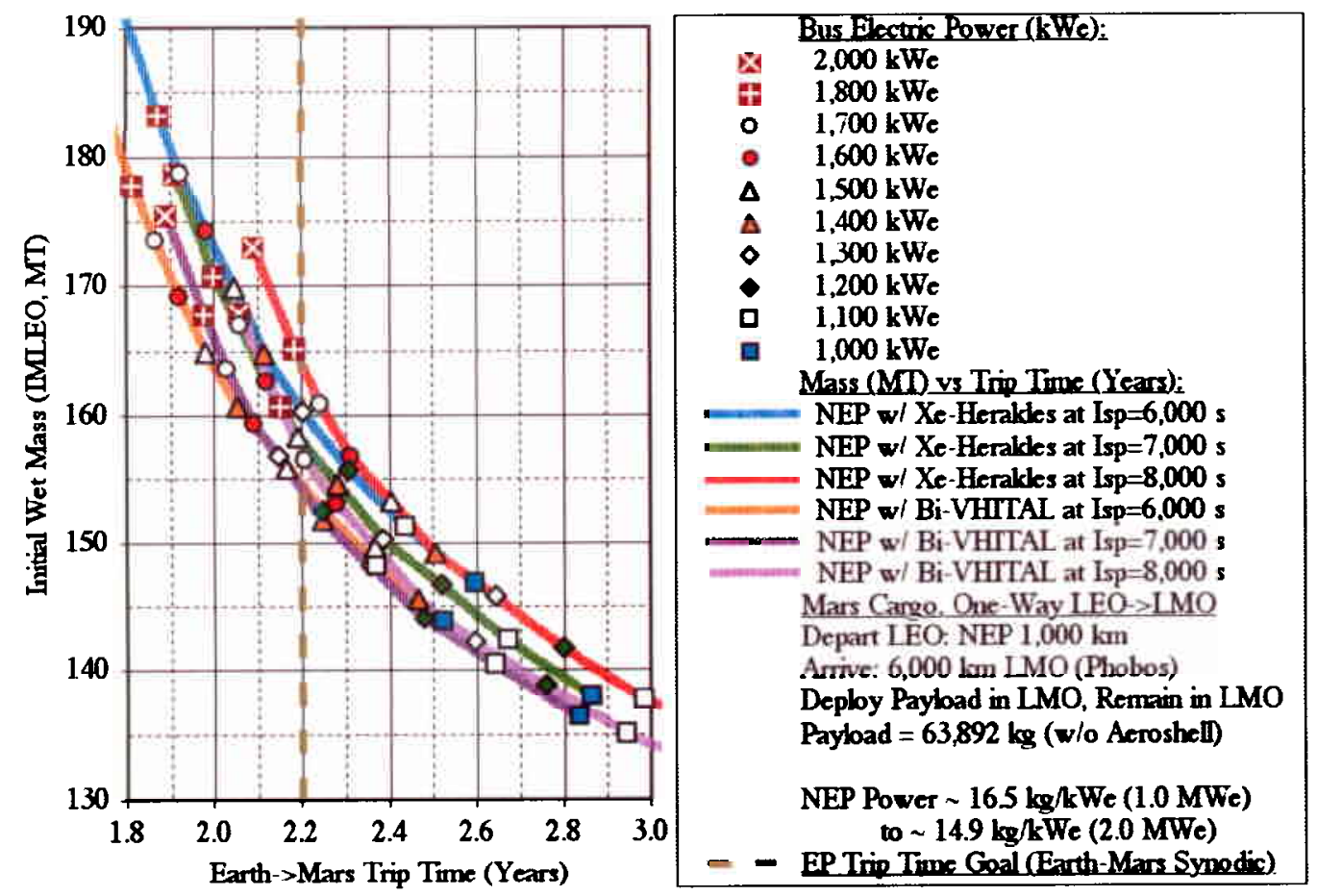

Figure 18. Variation in IMLEO and Trip Time for the NEP and SEP Mars Cargo Mission.

\section{Support System Development}

\section{Feed System}

Two feed systems are under development for the VHITAL-160 testing. TSNIIMASH is responsible for delivering a previously developed and demonstrated feed system. This system is robust and low risk; however it does not include a flow sensor and relies on a hydrostatic pressure feed that is not flight compatible. Bulk flow rate measurements are achieved by monitoring tank mass changes. This feed system is shown in Figure 19. The bismuth is delivered to the thruster through the vapor tube shown in the top of this figure. Figure 2 shows its mounting configuration.

The second feed system is under development at JPL, NASA MSFC and ESLI. The prototype feed system configuration is shown in Figure 20. It will be used to demonstrate and calibrate the feed system. NASA MSFC is responsible for the liquid phase of this system which will employ a gas pressurized stainless steel tank, stainless steel tubing, an electromagnetic

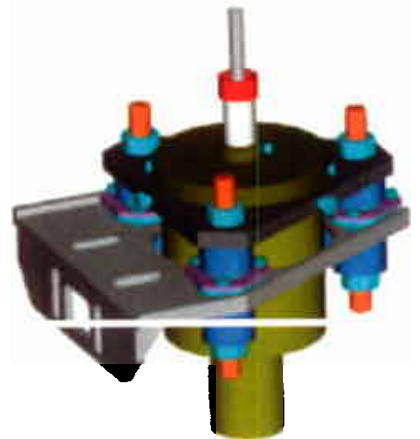

Figure 19. CAD drawing of the bismuth feed system developed by TsNIIMASH. (EM) pump and a heat pulse flow sensor. EM pumps are very robust, containing no moving parts and allow for the

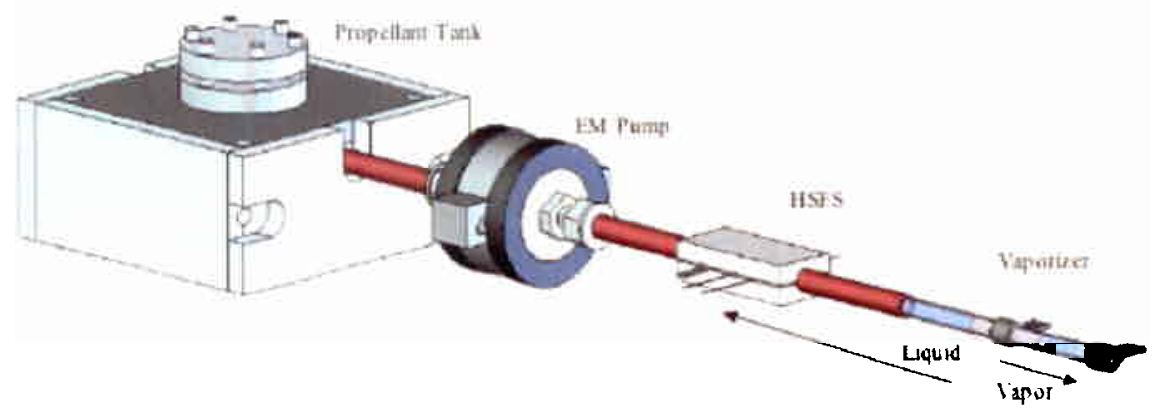

Figure 20. The prototype feed system configuration. 
isolation of the bismuth metal inside the feed line so that the pumping action can be attained without having to bring additional materials into contact with the fluid. Figure 20 shows a schematic illustration of our prototype bismuth EM pump design. The pressure developed is a function of the pump geometry, magnetic field strength, and current. With the required flow rates, the Re number will be low enough for the pump flow to be laminar: negligible viscous pressure drop is anticipated. The magnetic Reynolds number is very small for our operating conditions, which means that the flow will not be retarded by the dragging of magnetic field lines downstream (that is, the magnetic flux will not be "frozen" in the fluid). The main power loss in the pump will be Ohmic dissipation (Joule heating) in the electrodes. This power is expected to be $\sim 1 \mathrm{~W}$. Prototypes of these pumps have been fabricated at MSFC and demonstrated already on gallium.

A hotspot flow sensor (HSFS) is under development for flow rate measurements. An illustration of the sensor is shown in Figure 22. A pulse of thermal energy (derived from a current pulse and associated joule heating) is applied near the inlet of the sensor. This thermal feature (they are "tagging" the flow with a thermal feature) is convected downstream by the flowing bismuth. A downstream thermocouple records a "ripple" in the local temperature associated with the passing "hot-spot" in the propellant. By measuring the time between the upstream generation and downstream receiving of the thermal feature, the flow speed can be calculated using a "time of flight" analysis. The primary advantage of this technique is that it doesn't depend on an absolute measurement of temperature but, instead, flow thermal features are observed, which makes the technique insensitive to other system thermal fluctuations. The hotspot in the upstream flow is generated by pulsing current directly across the bismuth channel flow; by doing so we exploit the high resistivity of bismuth, and obviate the need for a separate resistive heating element. In order for the HSFS to provide useful results, the spatial integrity of the hotspot must be maintained until it reaches the thermocouple location. The hotspot will tend to "flatten out" as it propagates, due to thermal diffusion. Therefore we must design the device such that the thermal diffusion time scale is much smaller than the convective time scale. In our present design the convective time scale is $\sim 1 \mathrm{sec}$, whereas the (calculated) diffusion timescale is $\sim 15 \mathrm{sec}$, so we expect to convect a resolvable feature past the thermocouple position. The current pulse $(2 \mathrm{~A}, 1 \mathrm{msec})$ is expected to induce a local temperature rise of $\sim 10{ }^{\circ} \mathrm{C}$. Prototypes of the flow sensor have been fabricated and testing is underway at MSFC.

The vapor phase of the feed system employs a resistively-heated carbon vaporizer tube, a carbon fiber porous plug and will possibly include a propellant isolator. The porous plug vaporizer was demonstrated with electric propulsion in a zero gravity mercury-fueled ion thruster feed system [5]. The design of the vaporizer is a highly constrained problem because of the low vapor pressure of bismuth, shown in Figure 23, and the desire for minimal operating temperatures and pressures. Also, the plug and tube wall material at the plug must not be wettable by bismuth to prevent liquid flow through it. The desired bismuth vapor pressure entering the thruster is a few Torr. The EM pump for the liquid entering the thruster will require 1 A/Torr. To minimze the current requirement on the pump for a more desirable system, the pressure drop through the porous plug and the vapor pressure must be minimized. The pressure of the liquid entering the vaporizer must be greater than the vapor pressure at the vaporizer temperature and less than the sum of the capillary pressure and the vapor pressure at the vapor front: 


$$
P_{\text {vapor }}\left(T_{\text {iniet }}\right)<P_{\text {inlet }}<P_{\text {vapor }}\left(T_{\text {inlet }}\right)+P_{\text {surfitension }}
$$

The vapor pressure of bismuth can be represented by the following equation [6] and illustrated by Figure 24 with $\mathbf{P}$ in Pascal and temperature in Kelvin.

$$
P_{v}=\log ^{-1}\left(13.317-\frac{10114}{T}-0.86 \log T\right)
$$

The capillary pressure can be represented by the following relationship with capillary diameter, $D(\mathrm{~cm})$, and temperature sensitive surface tension coefficient [7], $\gamma$, for a non-wetting interface in the porous plug

$$
\begin{gathered}
P_{\text {surftesion }}=4 \gamma / \mathrm{D}, \quad \gamma=378 \mathrm{dyne} / \mathrm{cm}\left(271^{\circ} \mathrm{C}\right), \\
\mathrm{d} \gamma / \mathrm{dT}=-0.07 \mathrm{dyne} /{ }^{\circ} \mathrm{Ccm} .
\end{gathered}
$$

The flow rate of bismuth to the thruster is controlled by the vaporization rate of the bismuth in the vaporizer with vaporizer power. Each capillary channel in the porous plug will contribute to the total mass evaporation rate as defined by the Hertz-Knudsen equation

$$
\dot{m}_{c h}=\alpha_{v} m A_{e c h} \frac{P_{1}-P_{i m}}{\sqrt{2 \pi m k T}}
$$

where $\alpha_{\mathrm{v}}$ is the sticking coefficient of the bismuth vapors impinging on the liquid surface, $\mathrm{m}$ is the atomic mass of bismuth $(\mathrm{kg}), \mathrm{k}$ is the

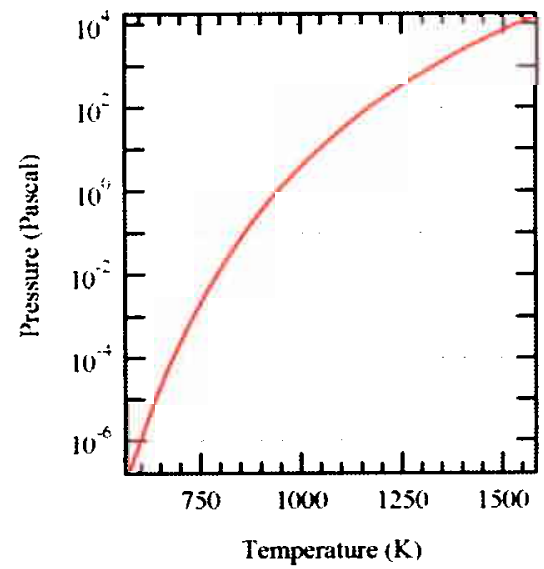

Figure 23. Bismuth vapor pressure. Boltzman constant, $A_{\text {ech }}$ is the surface area of the vaporizing liquid. The pressure at the evaporating surface, $P_{\text {in }}(\mathrm{Pa})$, which is the fluid surface in the each of the pores depends on the pressure at the exit of the pore channels, $P_{\text {out }}$, vaporizer temperature, pore radius, a $(\mathrm{m})$, atomic mass, $\mathrm{m}(\mathrm{kg})$, length of the pores, $\Delta z(\mathrm{~m})$. and mean free path in the pores, $\lambda(\mu)$, number density, $\mathrm{n}\left(/ \mathrm{m}^{3}\right)$, and dynamic viscosity, $\eta(\mathrm{kg} / \mathrm{m}-\mathrm{s})$, with the following relationship for the slip flow conditions that exist.

$$
\Delta P=P_{i n}-P_{\text {sut }}=-\frac{\frac{\dot{m}_{i k}}{n m} \frac{8 \eta}{\pi a^{4}}}{\left(1+\frac{8}{3} \frac{\lambda}{a}\right)} \Delta z
$$

Assuming parallel circular flow channels, which is an approximation to the true channel geometry that exists in the carbon fiber plug where the vapor will flow along the length of the fibers. The capillary diameter in this plug is characterized by a hydraulic diameter, which is the average diameter calculated from the ratio of the plug volume to area. With a feed system pressure requirement to the anode and flow rate requirement, we can estimate the total mass flow rate as a function of the number of channels and the mass flow rate per channel, $\mathrm{m}_{\mathrm{ch}}$,

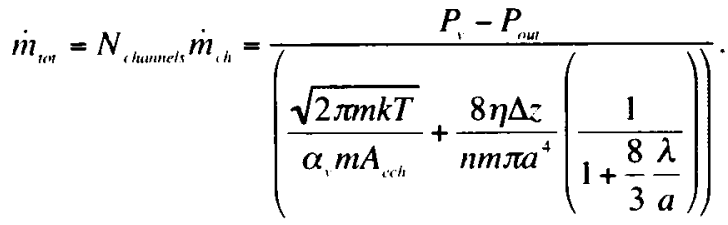

Axial and radial flow plug geometries were considered. The radial flow channels were preferred because of the more straightforward fabrication process. The plug geometry is cylindrical with radial oriented fibers so that the fluid flows along the inner diameter of the plug to a solid plug in the end and out radial as a vapor along the carbon fiber shafts. The model predicts the minimum flow rate expected as a function of vaporizer temperature. The temperature of the liquid is estimated by the vaporizer temperature. Based on this model of vaporizer performance, a carbon fiber porous plug vaporizer was designed with a resistively heated circular carbon exterior tube. The prototype vaporizer has been used to successfully demonstrate $2 \mathrm{mg} / \mathrm{s}$ bismuth evaporative flow rates at $1170^{\circ} \mathrm{C}(1443 \mathrm{~K})$. The vaporizer construction

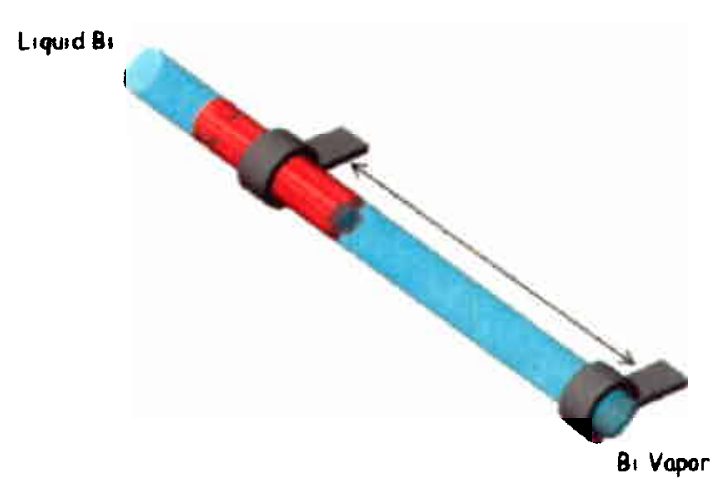

Figure 24. A CAD drawing of the all-carbon bismuth vaporizer fabricated at ESLI. 
has no joints, welds, brazes or CTE mismatch issues because the construction is entirely carbon with a carbon CVD process used to join the two components. Initial testing of the vaporizer will help to identify liquid pressure limitations and validate the model with pressure drop characteristics through the plug. Then minor plug geometry changes will be made to ensure $12 \mathrm{mg} / \mathrm{s}$ at $<1200{ }^{\circ} \mathrm{C}(1473 \mathrm{~K})$. Figure 24 shows the bismuth vaporizer configuration.

A gas pressurization system and tank for the bismuth were fabricated at NASA MSFC for characterizing the bismuth vaporizer. The stainless steel tank is surrounded by copper plates with embedded cartridge heaters in a macor box as shown in Figure 25. Bismuth is melted in the tank and then pressurized with a bang-bang regulation system that is shown in Figure 26. This system can control the liquid bismuth pressure to the vaporizer in the range of 0-200 Torr with $+/-2$ Torr accuracy. This system will be used to feed bismuth to the vaporizer, determine the maximum liquid pressure to the vaporizer and demonstrate the HPFS. Argon is used as the pressurizing gas.

\section{Test Facility}

The facilities will be employed for thruster testing. The TsNIIMASH facility will be employed for acceptance testing the thruster. The facility is shown in Figure 27. The experimental thruster technology assessment program will be carried out at JPL in a unique facility specially designed for testing electric thrusters operating on condensable metal

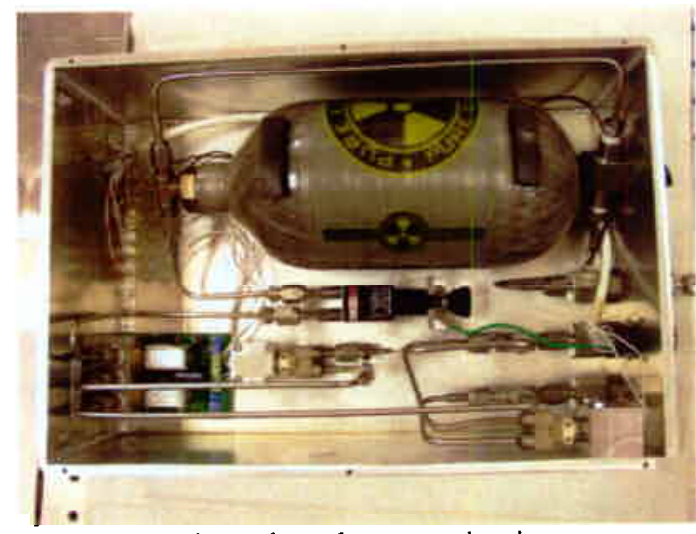

Figure 26. Bismuth tank pressurization system. propellants. The 3-m diameter, 8-m long vacuum chamber shown in Figure 28 contains a custom-fabricated liner designed to safely capture the high enthalpy Bismuth metal vapor plume. The cooled liner is capable of handling thermal loads up to $1.5 \mathrm{MWt}$. The facility is pumped by two $51 \mathrm{~cm}$ large oil diffusion pumps which have a pumping speed of $25,000 \mathrm{l} / \mathrm{s}$ on air. These pumps maintain a background pressure of $1.33 \times 10^{-4} \mathrm{~Pa}$ ( $10^{-6}$ Torr). The cooled liner serves as a Bismuth cryopump, and the condensed Bismuth metal layer on the liner will act as a getter, further reducing the pressure of residual gases in the facility. JPL's high power test facility currently has 1 MWe of electrical power available. The facility size, cooling capability and available electrical power are sufficient to support testing of condensable metal fueled thruster systems at power levels of over $1 \mathrm{MW}$. Carbon fiber beam targets will be employed to intercept the direct beam while the sidewalls will be lined with disposable aluminum foil to expedite the chamber cleaning process. The rigid carbon-carbon velvet panels, developed by ESLI, will significantly reduce the back flux of carbon and bismuth with a much lower mass in comparison to graphite panels.

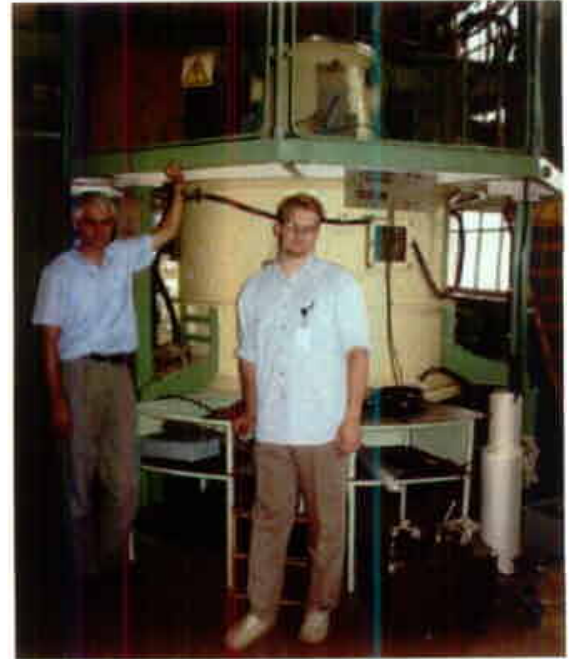

Figure 27. TsNIIMASH test facility for the bismuth fueled two stage Hall thrusters.

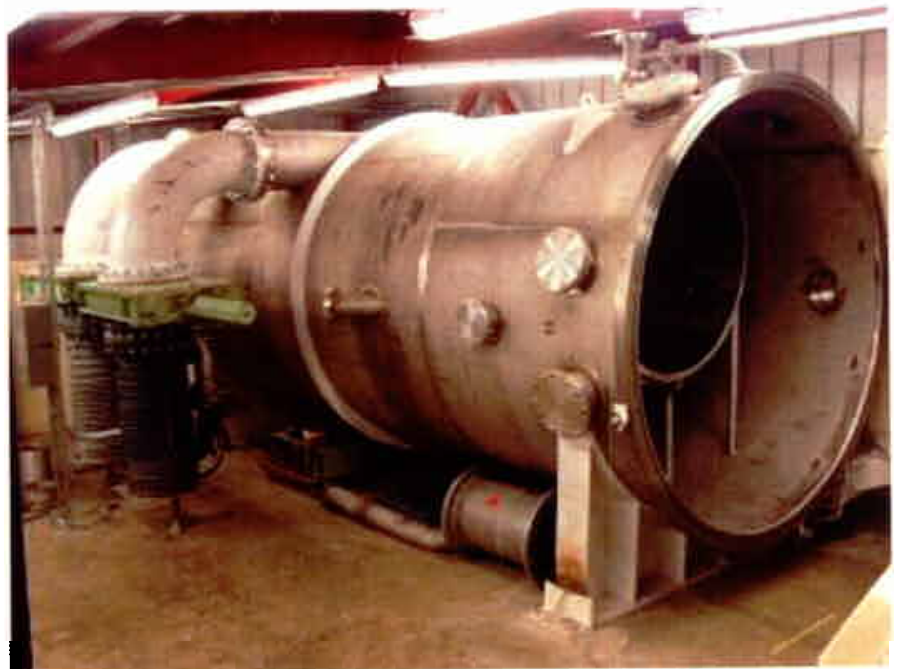

Figure 28. JPL liquid metal fed thruster test facility.

American Institute of Aeronautics and Astronautics 


\section{Performance Assessment}

\section{A. Experimental}

Experimental performance assessments of the VHITAL 160 will be conducted at TSNIIMASH and JPL. Thruster acceptance testing will be executed at TsNIIMASH to demonstrate the performance objectives at $25 \mathrm{~kW}(6000 \mathrm{~s})$ and $36 \mathrm{~kW}(8000 \mathrm{~s})$ before shipment to JPL. The refurbished TAL 160 will be tested at Ts.VIIMASH to demonstrate the support systems including the vacuum chamber, feed system, power system, thrust stand and diagnostics. The thrust stand that will be employed at TsNIIMASH is a vertical system that is externally mounted to the chamber flange behind the thruster, as shown in Figure 29. Performance measurements at

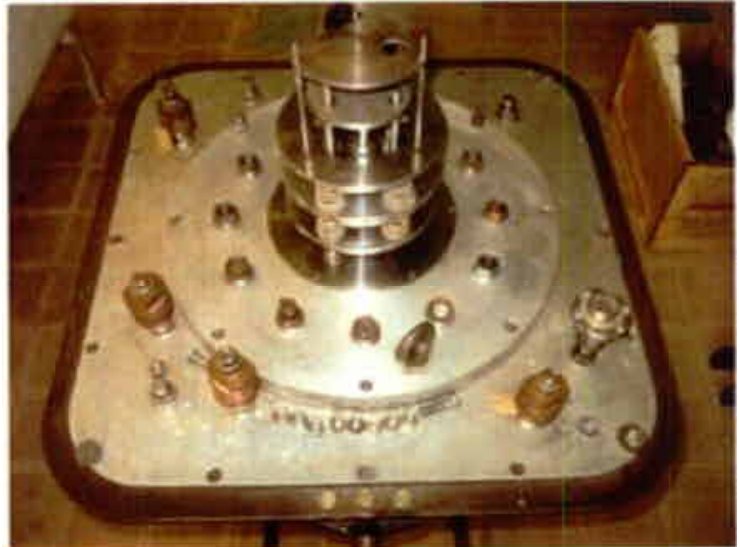

Figure 29. The vertical thrust stand at TsNIIMASH. JPL will be conducted at $25-36 \mathrm{~kW}$ and $6000-8000$

s. The thrust of the Bi-TAL will be measured using an existing thrust stand which is based on the NASA-GRC inverted pendulum thrust stand design [8] and was built to support $30 \mathrm{kWe}$ ammonia arcjet testing. It is capable of providing thrust measurements with an uncertainty of less than $2 \%$. It will be modified to support the heavier TAL and accommodate a liquid metal propellant and high voltage leads.

\section{B. Theoretical}

Theoretical performance assessment of the thruster will be lead by the University of Michigan. Previously, a hydrodynamic model of the second acceleration stage of the thruster was developed. [9] Recently, efforts are being concentrated on development of a hydrodynamic model of the first ionization stage that will be coupled with the second-stage model. To this end, a $2 \mathrm{D}$ hydrodynamic model of the first stage of the VHITAL-160 is under development. The diffuse form of the discharge in the first stage has been studied. This form of the discharge is sustained by the acceleration stage according to previous experiments. Therefore, the model of the first stage is coupled with the acceleration stage model [9]. At this preliminary point, the initial electron temperature is assumed as a downstream boundary condition.

In this model, we consider the plasma flow, which starts at the anode of the first stage. Quasi-neutrality is assumed and therefore the plasma presheath-sheath interface is considered as the lateral boundary for the plasma flow region. This model was described in detail elsewhere [10]. Here we just briefly review the main points of the model. We employ a hydrodynamic model in the $2 \mathrm{D}$ domain assuming that the system reaches a steady state. Only the radial component of the magnetic field is considered. The momentum and mass conservation equations for electrons, ions and neutrals under such conditions have the following form:

$$
\begin{gathered}
n m_{i}\left(V_{i} \nabla\right) V_{i}=n e E-\nabla P_{i}-\beta n m_{i} n_{a}\left(V_{i}-V_{a}\right) \\
\nabla \cdot\left(V_{i} n\right)=\beta n n_{a} \\
\nabla \cdot\left(V_{a} n_{a}\right)=-\beta n n_{a} \\
0=-e n(E+V \times B)-\nabla P_{e}-n v_{e f} m_{e}\left(V_{e}-V_{i}\right)
\end{gathered}
$$

where $n$ is the plasma density, $\beta$ is the ionization rate, $n_{a}$ is the neutral density, $V$ is the velocity, and $v_{e f}$ is the effective collision frequency.

Further model simplification includes consideration only of the one-dimensional flow of the neutral gas atoms. Finally, electron transport is considered in a one-dimensional framework along the channel median. Since only the radial magnetic field component is considered, the electron transport is much greater in the azimuthal direction $(\mathrm{E} \times \mathrm{B}$ drift) than in the axial direction (drift diffusion due

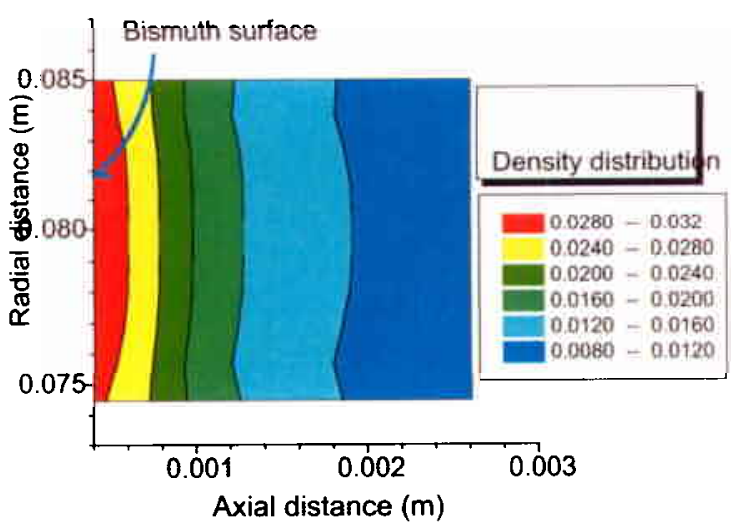

Figure 30. Plasma density distribution in the first stage of the VHITAL-160. 
to collisions). We consider that electron transport across the magnetic field is due to several collision mechanisms: electron-neutral collisions and anomalous (Bohm) diffusion: $v_{e f}=v_{e n}+v_{B}$, where $v_{e t}$ is the effective electron collision frequency.

The model includes current continuity analysis at the anode. Discharge current in the range of 4-6 $\mathrm{A}$ is considered. It is found that bismuth ionization is very efficient and occurs in the vicinity of the evaporated propellant surface. Figure 30 shows the computed plasma density distribution in the VHITAL-160 first stage. The current-voltage characteristics of the first stage are shown in Figure 31. The computed results are found to be in good agreement with data measured for this thruster at TSNIIMASH.

\section{Lifetime Assessment}

\section{Experimental}

Stanford University is responsible for the thruster lifetime assessment. The throughput demanded under the original NRA necessitates the implementation of new diagnostics approaches to validate the projected life of the VHITAL 160 thruster. The primary life-limiting mechanism is the erosion of the molybdenum (Mo) guard rings. One of the primary objectives of the Phase I research was to develop a plan to characterize the ion energy (using Laser Induced Fluorescence - LIF), and the ion flux impinging on the guard ring. These measurements will be conducted by Stanford University. Together with independent measurements of the ion-energy and angular dependent sputter yields that will be carried out at Colorado State University in Phase II of the program, these measurements will be used to predict the erosion behavior to end-of-life. Also during Phase II-IIl, our collaborators from CSU will develop and apply Cavity Ring Down Spectroscopy (CRDS) measurements for characterizing the Mo erosion products present in low concentrations in the vicinity of the exit of the thruster. While the CRDS measurements together with a simple Monte-Carlo model for erosion product species flow can further validate these erosion predictions, the CRDS measurements will be calibrated against Surface Layer Activation (carried out by the JPL team) which will allow a more direct measurement of the instantaneous rate of ion-induced erosion at the two identified operating points. CRDS will then be used for studying the erosion behavior outside of these selected operating points.

\section{Laser Induced Fluorescence}

During Phase I, we completed an extensive analysis of the spectroscopic lineshapes of bismuth ions with attention focused on identifying relatively strong spectral lines (transitions) in the near IR (roughly 700-900 $\mathrm{nm}$ ) that are desirable for velocity measurements for their accessibility via laboratory laser systems. The subset of lines accessible to off-the-shelf tunable diode lasers are preferred for these LIF measurements because of the transportability to JPL. In order to make accurate velocity (energy) and energy distribution measurements, an understanding of the lineshapes of these transitions is required. During Phase 1, an extensive analysis of the spectroscopic lineshapes of bismuth ions was completed.

For purposes of number density determination of Bil, the line at $306.86 \mathrm{~nm}$, which is coupled to the ground state, will likely be used in atomic resonance absorption spectroscopy, which can incorporate a measurement of the Lorentzian broadening if experimental resolution is high enough to detect the lineshape. A resonant transition for Bill lies at $143.68 \mathrm{~nm}\left(6 \mathrm{p}^{2}{ }^{3} \mathrm{P}_{0}-7 \mathrm{~s}{ }^{3} \mathrm{P}_{1}\right)$; note that this line is a forbidden transition since $\Delta L=0$, but it has been recorded successfully with high intensity [11]. For the neutral line, a Bi hollow-cathode lamp can be used as a broadband lightsource; other options, such as broadband absorption in the ultraviolet, would need to be developed for the ion line. In the near IR region, the candidate transitions of interest for velocity measurements of $\mathrm{Bil}$ are at $784.25 \mathrm{~nm}\left(6 \mathrm{p}^{3}{ }^{2} \mathrm{P}_{3 / 2}-7 \mathrm{~s}^{2} \mathrm{P}_{1 / 2}\right)$ and $854.7 \mathrm{~nm}\left(6 \mathrm{p}^{3}{ }^{2} \mathrm{P}_{3 / 2}-7 \mathrm{~s}{ }^{4} \mathrm{P}_{3,2}\right)$; both of these lines are forbidden due to $\Delta L=0$, but have been recorded and analyzed successfully [12]. Candidate transitions for velocity measurements of Bill are at $796.7 n m\left(7 p^{3} D_{2}-8 s{ }^{3} P_{1}\right)$ and $854.3 n m\left(7 p{ }^{3} D_{2}-6 d{ }^{3} P_{1}\right)$. These near IR lines are relatively strong, reachable with off-the-shelf tunable diode lasers, and have available hyperfine splitting constants in the literature [11,12]. 
When taking measurements on a Hall thruster like the VHITAL system, the hyperfine peaks are expected to be broadened to form a more continuous lineshape. An analysis of the causes of this broadening is necessary for thruster diagnostics.

Having identified suitable transitions in $\mathrm{Bi}$ (such as the $854.7 \mathrm{~nm}$ line) and Bill (such as the $854.3 \mathrm{~nm}$ line) for LIF velocimetry measurements in the VHITAL 160 , we proceeded to initiate an experimental program aimed at developing practical experience in carrying out LIF and/or absorption on these transitions using an available tunable Coherent (Model VERDI) YAGpumped (Coherent Model 899) Ti:Sapphire laser. This laser is appropriate for laboratory assessment and surveys of spectral lines over a broad range of wavelengths. However, once experimentally verified, we intend to transition to an external cavity tunable diode laser, that is tunable over approximately $30 \mathrm{GHz}$, about a pre-selected wavelength.

In order to progress with work on the bismuth spectrum necessary for the development of optical diagnostics for the V'HITAL project, a bismuth heatpipe vapor cell has been developed. A bismuth sample is heated inside the chamber, producing a vapor for absorption analysis using a Deuterium lamp as the broadband light source. A hollow cathode discharge inside the chamber is used to generate a bismuth plasma for analysis in emission and absorption. Further, the cell has been constructed with suitable optical ports for LIF analysis of neutral and ionized bismuth. With this chamber, specific candidate transitions will be analyzed to further develop the bismuth neutral number density diagnostic, as well as to verify the lineshape models for, and the accessibility of, the candidate transitions for LIF measurements.

Additionally, to continue with diagnostic development beyond the capabilities of the stationary gas in the heat-pipe apparatus, an SPT thruster is being modified with a bismuth propellant feed system to generate a high velocity bismuth plasma. The existing thruster is being fitted with an electrically heated bismuth vaporizer cell coupled to a modified anode to inject bismuth into the channel. The high velocity ions produced by this thruster will be analyzed to further develop the bismuth diagnostics specific to a moving plasma, as well as to aid in the selection of a diode-laser system for use with the VHITAL thruster.

A detailed design for the LIF velocimetry measurements to be carried out at JPL during Phase III of the program has been carried out. The measurements will be made using two external cavity diode lasers tuned to the Bil and Bill transitions specified above. A large $(4 \mathrm{ft} \times 8 \mathrm{ft}$ ) table adjacent to the vacuum chamber supports the majority of the optical components used in this diagnostic. The output of the laser passes through an optical isolator (to prevent reflected light from returning into the laser) and is then split into five beams, three of which are to monitor the absolute wavelength (with the wavemeter), output laser intensity (photodiode), and the wavelength scanning rate (Fabry-Perot interferometer). The remaining two beams serve as the radial and axial pump beams. The radial pump beam is directed vertically and over the top of the chamber to the overhead window, and 
then directed vertically downwards so that it propagates along the radial direction when it is centered about the axis of the thruster. The "axial" beam stays in the horizontal plane of the thruster directed into the chamber through a port at an oblique angle with the thruster axis. In this way, it samples a component of the axial velocity of the directed molecules, which, together with the radial component completes the desired radial/axial vector velocity field. Each beam is appropriately chopped and the LIF signal from a common excitation volume is collected by an optical train through a side port at ninety degrees from the thruster axis, and analyzed using a monochromator filter and photomultiplier combination. Multiplex phase-sensitive detection allows the collection of both fluorescence signals using a single detection system by chopping the two pump beams at different frequencies.

\section{Surface Layer Activation}

The SLA technique will be used to calibrate the CRDS technique at the two critical operating points by directly measuring the mass loss. A thin surface layer in the guard rings will be labeled with a radioactive tracer. As the surface erodes, the activity level decreases. With a calibration of activity level vs. eroded depth, the erosion rate can be determined from the decrease in activity during operation. SLA provides highly sensitive (sub-micron resolution), temporally and spatially resolved, non-intrusive in situ erosion measurements. It has been used to measure erosion in magnetoplasmadynamic (MPD) thrusters [13,14], hollow cathode orifice plates [15], wear in internal combustion engines [16] and turbines, corrosion in chemical plants, arcing damage in tokomaks, and wear in prosthetic hip joints. Layers up to tens of microns deep can be labeled with the tracers; thinner layers can be produced by bombarding the surfaces at an angle. Changes in activity level (erosion of the activated layer) on the order of $1 \%$ are detectable. With activations that have already been developed, erosion rates can be determined to within about $\pm 10 \%$ by running the thruster long enough to accumulate 2 microns of erosion.

If resources permit, both Mo and $\mathrm{C}$ guard ring segments will be activated to measure the erosion rates of both materials during the same experiments. Mo guard rings will be activated by bombarding it with a $10.4 \mathrm{MeV}$ proton beam. This results in two nuclear reactions with transmutes a small amount of the Mo ${ }^{95}$ to the gamma-emitting technetium isotopes, $\mathrm{Tc}^{95 \mathrm{~m}}$ and $\mathrm{Tc}^{96}$. The reaction yield for $\mathrm{Tc}^{96}$ is 60 times higher than $\mathrm{Tc}^{95 \mathrm{~m}}$; however, with a much shorter half-life (4.28 days), its activity level becomes negligible after approximately 2 months. The half-life of $\mathrm{Tc}^{95 \mathrm{~m}}$ is 58.72 days [15]. The erosion rate of Mo hollow cathode keepers was measured with a resolution of 0.2 um at JPL with this activation. This demonstrated approach to these measurements will be employed. Carbon guard rings will be activated by implanting Be- 7 into it. This tracer has a half-life of 53.28 days and can be used immediately for measurements. The gamma spectroscopy system that was used to detect surface activation decay rates in the keeper erosion rate measurements at JPL will also be used in these experiments.

\section{Cavity Ring Down Spectroscopy}

CRDS will then be used to quantify the changes in flux of erosion products from the thruster with changes in operating conditions over a broader range of thruster operation. CRDS is a highly sensitive laser-based absorption technique $[17,18]$ that is directly quantifiable and thus well suited for measurements of low concentrations of sputtered particles. It is an absorption technique, so that unlike fluorescence and emission measurements, ground states are measurable and there are no quenching interferences. As shown in Figure 28, the basic idea is to house the absorbing sample (i.e. the sputtered atoms of the thruster plume) within a high-finesse optical cavity formed from high-reflectivity mirrors ( $R \sim 0.9999$ ). The interrogating laser beam is coupled into the optical cavity where it "bounces" many times back-and-forth between the mirrors. Owing to the high reflectivity, the light within the cavity makes many $\left(\sim 10^{4}\right)$ passes within the cavity, and the effective path length and thus sensitivity is greatly increased. A detector placed behind the cavity measures the temporal decay of optical intensity within the cavity. The difference in the temporal decay rate with and without the absorber (or with the laser tuned on/off the resonance) yields the sample concentration. The technique has been used widely for the measurements of trace species in flames, plasmas, and the atmosphere, and has recently been applied to sputter measurements for electric propulsion applications $[19,20]$.

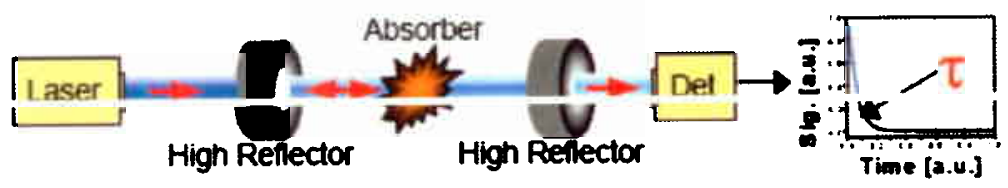

Figure 28. Schematic diagram of cavity ring-down spectroscopy (CRDS). 
The CSU team has developed a high sensitivity bench-top CRDS detection system $[19,20]$. The key components are an ion beam and target, housed within a vacuum facility. The molybdenum target is typically a circular or rectangular sheet (characteristic dimension $\sim 8 \mathrm{~cm}$ ). The set-up uses a broadly tunable optical parametric oscillator (OPO) laser system (doubled idler) as the light source to probe optical transitions in the $380-400 \mathrm{~nm}$ region. Spectroscopic models (including lineshape effects) are used to identify appropriate spectroscopic transitions, and to quantitatively analyze data. A partial energy level diagram for molybdenum is shown in Figure 29a. The CRDS detection scheme for Mo is based upon probing strong optical transitions in the $-370-400 \mathrm{~nm}$ region originating from the ground electronic state. Absorption lines at $379.93 \mathrm{~nm}, 386.52 \mathrm{~nm}$, and $390.41 \mathrm{~nm}$ have been measured. The areas of the measured absorption lines are fitted with Voigt peaks, the areas of which yield the path-integrated number density of the measured (ground) state. Figure $29 \mathrm{~b}$ shows the dependence of measured number density versus beam current. In order to provide representative concentration values, we have assumed a uniform concentration over a fixed path-length corresponding to the ion beam extent of $8 \mathrm{~cm}$. In principle one could spatially scan the beam and use an Abel inversion to obtain a radial concentration profile. The inset to Figure $29 \mathrm{~b}$ shows the measured $386.5 \mathrm{~nm}$ absorption feature collected at several beam currents.

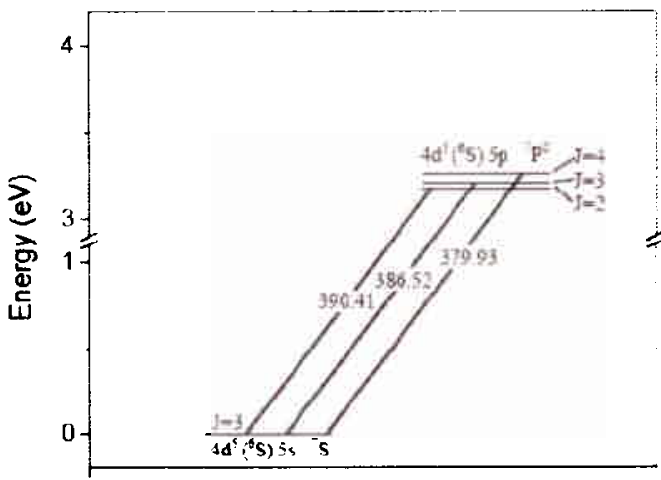

a)

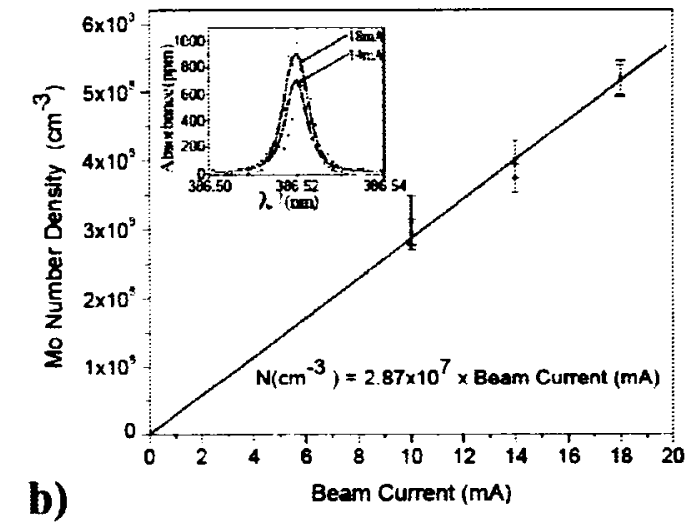

b)

Figure 29 a): Partial energy level diagram for molybdenum. b): Dependence of (average) molybdenum number density on beam current.

The sensitivity of the current CRDS bench-top system is significantly higher than the anticipated requirement for the VHITAL thruster. The noise level in the current system is equivalent to an absorbance of $\sim 2 \mathrm{ppm}$, or a pathintegrated concentration of Mo of $\sim 6 \times 10^{6} \mathrm{~cm}^{-2}$. The anticipated path-integrated value within the thruster is approximately $10^{8}-10^{9} \mathrm{~cm}^{-2}$. The linear dependence (through the origin) of concentration versus beam current, as well as results from our sputter model and taken as initial validation of the CRDS molybdenum measurements. The current measurement scheme is based on measuring the population of the Mo ground state. The possibility of significant fractions of the sputtered atoms being in elevated energy levels (as has been observed for certain species $[21,22])$ is currently under investigation.

The CSU team will extend the bench-top scheme summarized above to develop a system for implementation of the VHITAL thruster. Implementation of a high sensitivity CRDS system at the JPL vacuum chamber facility will pose several challenges associated with: the relatively long $(\sim 3 \mathrm{~m})$ optical axis of the JPL chamber, potential for cavity misalignment due to chamber pump-down and possible thermal expansion effects, and reduction of effective cavity finesse (and CRDS sensitivity) due to mirror contamination from condensed bismuth or sputter products. The CSU team is developing appropriate hardware and methodologies to meet these concerns. During Phase 3, CRDS measurements of sputtered molybdenum (from eroded guard-rings) will be performed as the thruster operating conditions are varied. These measurements will provide information on how the erosion varies with thruster operating conditions and will be used to validate computational models addressing thruster erosion. Although the CRDS will only measure molybdenum erosion (from Mo guard rings), other materials such as graphite or diamond are more likely candidates for the final guard-ring material. The CRDS measurements of Mo will contribute to validation of the overall erosion modeling and life assessment approach and thus will assist in the evaluation of all candidate materials.

With this multi-pronged approach to these measurements, a large trade space can be explored to determine the effect of operating voltage and current on thruster lifetime with low risk high fidelity measurements at the two critical operating points. This approach ensures that we can identify the lifetime at the critical operating points and the impact of critical operating parameters on thruster lifetime 


\section{Theoretical}

Theoretical lifetime assessment of the thruster will be conducted by the University of Michigan and Stanford University. The code developed with models of the plasma generation and acceleration zones will be used to predict the flux, energy, and incident angle of energetic particles impinging on the guard rings. Experimental measurements made in the near field of the thruster of the ion energies and fluxes will be used to validate the model. The sputter yields of the guard ring materials will be measured at CSU at the expected ion energies. The sputter yield measurements will be used with the code to estimate the erosion rates of the guard rings. The erosion rate model will be refined and validated with the experimental data. This model will be used to explore the thruster lifetime in operating regimes not supported by the experimental program.

A Hall thruster erosion model that has been developed at Stanford will also be applied in the lifetime predictions. These velocities will serve (i) as a means of directly indicating the velocity of ions impinging on the downstream edge of the guard ring, and (ii) as a means of validating the numerical simulations of the discharge, from which we can obtain the ion velocity distribution along the entire guard ring. Along with a measure of the ion current at the downstream edge of the guard ring using an ion probe flush with the guard ring surface (to be carried out in Phase III), and with known angular and speed (energy)-dependent sputter yields for $\mathrm{Bi}$ on $\mathrm{Mo}$, (i) provides the needed information for estimating the initial guard ring erosion rates. The validated simulations provide the necessary information for carrying out the initial erosion rates along the entire guard ring surface and for computing the evolution of the guard ring erosion pattern to end-of-life. During Phase I, we have developed this method of using simulations for estimating the initial erosion behavior of a Hall discharge of the stationary plasma thruster type. An SPT-like thruster was used as a test bed for carrying out these Life Assessment Analyses because of the availability of a Stanford-developed 2-D hybrid model of an SPT-like thruster, and because of the availability of sputter-yield data for xenon ions on BN. During Phase Il, the development of similar models for TAL thrusters, and collection of the sputter yield data for bismuth on molybdenum will allow an extension of these analyses to the VHITAL-160.

A detailed description of the 2-D hybrid Hall thruster model is described in Ref. 23. The numerical model treats the electrons as a quasi-one-dimensional fluid and the ions and neutrals as discrete particles advanced in physical space using a Particle-in-Cell (PIC) approach. The electrons obey a generalized form of Ohm's law, and a transient, spatially varying electron temperature profile is calculated at each time step through solution of the electron energy equation. In the momentum and energy equation an experimentally-based effective mobility is imposed to account for the anomalously high electron transport across magnetic field lines. Actual sputter yield data will be used in the model once it becomes available. This model was applied to a $200 \mathrm{~V}$ discharge hall thruster and successfully predicted thruster erosion rates. The simulation cannot yet predict the continuous erosion behavior, although a simple model is under development based on a method similar to that discussed in Ref. 24 . This simple model accounts for the changing incident ion angle with the receding surface as erosion proceeds with time. It also accounts for the possibility of ion scattering off of neutrals in the vicinity of the discharge wall. Results of this model are forthcoming.

\section{Spacecraft Contamination Potential Assessment}

\section{E. Experimental}

The plume contamination assessment will consist of measurements of both the neutral bismuth density and velocity, which can serve as a starting point for propagating the plume to the far-field using numerical simulations and current density distribution measurements to validate the simulations. Faraday probes will be used for the current density distribution measurements. LIF velocimetry will be applied for neutral bismuth and bismuth ion velocity measurements and absorption spectroscopy will be implemented for neutral bismuth density measurements. The transition in Bil for LIF Velocimetry has been identified in the phase I effort. Spectral absorption and sensitivity analysis associated with the Bil resonance transition was conducted and the results are included in the experimental lifetime assessment section.

\section{F. Theoretical}

The contamination potential assessment effort will be lead by the University of Michigan. The program includes a plan to develop a model of the plasma flow from the thruster and a model to track the expansion of the exhaust and backflow of condensable species onto spacecraft surfaces. Output from the modeling of the plasma generation region will provide boundary conditions for the separate computation of the plasma plume. This separation is desirable because there are many physical phenomena important in the device flow that are negligible in the plume (e.g., magnetic field effects). The plume computations can be numerically expensive as they must be conducted over a large spatial domain and, thus, it is important to simplify the plume modeling as much as possible. Information for 
the atoms and ions will be communicated from the device model to the plume model via velocity distribution functions. This approach has been successfully applied in the end-to-end modeling of the SPT-100 xenon-fueled Hall thruster.

The plasma plumes of both stationary plasma (SPT) and anode layer (TAL) Hall thrusters using xenon propellant has been modeled numerically in a number of studies $[25,26,27,28]$. Most of these models employ a particle approach similar to that proposed for the device model. The electrons will be modeled as a fluid, whereas the ions (both $\mathrm{Xe}^{+}$ and $\mathrm{Xe}^{2+}$ ) and neutral atoms are modeled as particles. The ions are subsequently accelerated in the electric fields in a self-consistent manner. Detailed models of the relevant collision processes to be used in this model have been developed and successfully applied to predict Hall thruster plume characteristics measured in the lab [29] and in space [30].

Prediction of contamination depends on the number flux of $\mathrm{Bi}$ to the guard rings together with a model for surface-absorption processes. Since $\mathrm{Bi}$ is highly condensable, it is reasonable to assume a sticking coefficient of one. The guard ring surface erosion rate will be predicted from the flux, energy, and incident angle of ions and models and measurements for the sputter yields of the materials of interest. These models will be developed based on semi-analytical approaches as reviewed by Boyd and Falk [31].

In recent progress a new, general $2 \mathrm{D} / 3 \mathrm{D}$ PIC-DSMC hybrid code using unstructured meshes is being employed [32] to model the thruster plume. Both charge exchange and momentum exchange collisions are included in the simulation. The plume simulation begins at the exit plane of the thruster using output from the model of the second stage of the thruster [9]. Figure 30 shows contours of plasma density for an accelerating current of $3 \mathrm{Amp}$ and a mean accelerating voltage of $5 \mathrm{kV}$. Measurements of the ion current density were taken at TSNIIMASH in the 1980's at this operating point, and Figure 31 compares the results of a preliminary simulation with the measured data to demonstrate progress on this component of the modeling program. Parametric studies will be performed to try to improve the agreement between these data sets.

\section{Summary and Conclusions}

The VHITAL Program has progressed 12 months into the 30 month program towards the evaluation of this two stage bismuth-fueled Hall thruster technology for high power NEP missions. TsNIIMASH finished the design analysis and design documentation of the VHITAL-160 and proceeded to thruster fabrication. The TAL-160 has been refurbished and performance demonstrations are underway for the thruster and support systems at 
TsNIIMASH. NEP mission studies have been completed for robotic missions to Mars, 200 A.U. and Saturn. The results showed that this technology has the potential to be very attractive and competitive with existing propulsion options for these missions. Significant savings in trip time, mass and system complexity could be realized because of the high power, high specific impulse and low bismuth propellant tankage fractions relative to more conventional xenon fueled ion engines. Significant progress has been made on both feed systems under-development. The TsNIIMASH feed system is operational in TAL-160 testing. The prototype porous plug demonstrated functionality as a liquid bismuth plug and vaporization of $2 \mathrm{mg} / \mathrm{s}$ of bismuth with an all carbon two-component design. A hydrodynamic model of the first stage of the discharge is under development to couple with the existing hydrodynamic model of the second stage. Preliminary results show good agreement with experimental results. Lifetime models and diagnostics are under development with significant progress. A heat-pipe vapor cell was developed as a bismuth source to use in the development of the bismuth neutral number density diagnostic and to verify the lineshape models for candidate transitions for the LIF diagnostic on bismuth ions and neutrals. The detailed diagnostic approach and required equipment have been identified. The 2D/3D PIC-DSMC hybrid code has been applied to model the thruster plume using experimental data from TsNIIMASH with preliminary results.

\section{Acknowledgments}

The research described in this paper was carried out at Stanford University, the Jet Propulsion Laboratory, California Institute of Technology, the University of Michigan, Colorado State University, NASA MSFC, and Energy Science Laboratories. The authors would like to thank Dr. Gani Ganipathi for assistance with the feed system development, Dr. Ira Katz for the cathode plume analysis and Kelly Stephanie for creating the feed system diagram. The research was conducted under a contract with the National Aeronautics and Space Administration. The authors would like to thank John Warren in Prometheus Advanced Systems and Technology Office and Ron Reeve in the Spacecraft Propulsion Technology, Space Exploration Technology Program for support of this work.

\section{References}

1. Manzella, D. Jacobson, and D. T., Jankovsky, "High Voltage SPT Performance," AIAA Paper 2001-3774.

2. Tverdoklebov, S., Semenkin, A., Polk, J., "Bismuth Propellant Option for Very High Power TAL Thruster," AIAA Paper 2002-0348.

3. Noca, Muriel, NASA JPL, Personal Communication, 2003.

4. Fiehler, D., et al., "Electric Propulsion System Modeling for the Proposed Prometheus 1 Mission," AIAA Paper AIAA 2005-3891, Presented at the 41st AIAA/ASME/SAE/ASEE Joint Propulsion Conference, Tucson AZ, 1013 July, 2005.

5. Kerrisk, D. J., Masek, T. D., "A Zero Gravity Mercury Propellant Feed System," AIAA Paper 66-250.

6. Massey, D., Kieckhafer, A., Sommerville, J., King, L., "Development of a Vuporizing Liquid Bismuth Anode for Hall Thrusters," AIAA Paper 2004-3768.

7. Beer, S.Z. (ed.), Liquid Metals Chemistry and Physics, Marcel Dekker, Inc., 1972, pp. 165-169

8. Haag, T., "Design of a Thrust Stand for High Power Electric Propulsion Devices," AIAA Paper 89-2829.

9. M. Keidar, I.D. Boyd and I.I. Beilis, "Modeling of a high-power thruster with anode layer," Physics of Plasmas, 11, No. 4, 2004, pp. 1715-1722.

10. M. Keidar, I.D. Boyd and I.I. Beilis, "Plasma flow and plasma-wall transition in Hall thruster channel," Physics of Plasmas, 8, No. 12, 2001, pp. 5315-5322.

11. Dolk, L., Litzen, U., and Wahlgren, G.M., "The Laboratory Analysis of Bi II and its Application to the Bi-rich HgMn Star HR 7775," Astrononmy \& Astrophysics 388, 2002, pp. 692-703

12. George, S., Munsee, J.H. and Vergès, J., "Hyperfine-structure Measurements in Bismuth Using a Fouriertransform Spectrometer" J. Opt. Soc. Am. B/Vol. 2, No. 8. August 1985, pp. 1258-1263.

13. Polk, J. E., Kelly, A. J. and Jahn, R. G., "Characterization of Cold Cathode Erosion Processes," AIAA Paper 88075.

14. Conlon, T. W., "Doping Surfaces with Radioactive Atoms - For Research and Industry," Contemp. Phys., 23(4), pp. 353-360, 1982.

15. Kolasinski, R.D., Polk, J.E., "Characterization of Cathode Keeper Wear by Surface Layer Activation," Journal of Propulsion and Power, Vol. 20, No. 6, 2004, pp. 992-999. 
16. Blatchley, C. C. and Sioshansi, P., "Surface Layer Activation Technique for Monitoring and In Situ Wear Measurement of Turbine Components," Jet Propulsion, 3(3), pp. 248-252, 1986.

17. K.W. Busch and M.A. Busch, Cavity-Ringdown Spectroscopy (ACS Symposium Series 720, Oxford University Press, 1999).

18. G. Berden, R. Peeters, and G. Meijer, "Cavity Ring-Down Spectroscopy: Experimental Schemes and Applications," Int. Reviews in Physical Chemistry 19(4), 565-607 (2000).

19. V. Surla, P.J. Wilbur, J.D. Williams, M. Johnson. A.P. Yalin, "Sputter Erosion Measurements of Titanium and Molybdenum by Cavity Ring-Down Spectroscopy", Review of Scientific Instruments, 75, 9, pp. 3025-3030 (2004).

20. A.P. Yalin, V. Surla, M. Butweiller, J.D. Williams, "Detection of Sputtered Metals using Cavity Ring-Down Spectroscopy", accepted for publication in Applied Optics (May 2005).

21. C. Staudt, and A.Wucher, "Sputtering of Ag atoms into metastable excited states," Phys.Rev. B 66,085415 I12(2002).

22. J. Bastiaansen, V. Philipsen, P. Lievens, R.E. Silverans, E. Vandeweert, "Influence of the atomic structure on the quantum state of sputtered Ir atoms" Phys. Rev. A 70, 052902 (2004).

23. E. Fernandez, M. Cappelli, and K. Mahesh, NASA/Stanford Center for Turbulence Research, Annual Research Briefs, pp. 81-90, 1998.

24. George, S., Munsee, J.H. and Vergès, J., "Hyperfine-structure Measurements in Bismuth Using a Fouriertransform Spectrometer" J. Opt. Soc. Am. B/Vol. 2, No. 8, August 1985, pp. 1258-1263.

25. Oh, D. Y., Hastings, D. E., Marrese, C. M., Haas, J. M., and Gallimore, A. D., "Modeling of Stationary Plasma Thruster-100 Thruster Plumes and Implications for Satellite Design," Journal of Propulsion and Power, Vol. 15., No. 2, 1999, pp. 345-357.

26. Boyd, I. D., "Computation of the Plume of an Anode Layer Hall Thruster," Journal of Propulsion and Power, Vol. 16, 2000, pp. 902-909.

27. Boyd, 1. D., "Review of Hall Thruster Plume Modeling." Journal of Spacecraft and Rockets, Vol. 38, 2001, pp. 381-387.

28. Boyd, I. D., "Hall Thruster Far Field Plume Modeling and Comparison to Express Flight Data," AIAA Paper 2002-0487.

29. Katz, 1. et al., "A Hall Effect Thruster Plume Model Including Large-Angle Elastic Scattering," AIAA Paper 2001-3355.

30. Boyd, I. D., "Hall Thruster Far Field Plume Modeling and Comparison to Express Flight Data," AIAA Paper 2002-0487.

31. Boyd I.D. and Falk, M.L., "A Review of Spacecraft Material Sputtering by Hall Thruster Plumes," AlAA Paper 2001-3353.

32. Cai,C. and Boyd, I.D., "3D Simulation of Plume Flows from a Cluster of Plasma Thrusters," AIAA Paper 20054662, June 2005. 